\title{
Oxidation induced restructuring of Rh-Ga SCALMS model catalyst systems
}

\author{
Haiko Wittkämper ${ }^{1,+}$, Sven Maisel ${ }^{2,+}$, Mingjian Wu ${ }^{3}$, Johannes Frisch ${ }^{4,5}$, Regan G. Wilks, ${ }^{4,5}$ Mathias \\ Grabau $^{1}$, Erdmann Spiecker ${ }^{3}$, Marcus Bär ${ }^{1,4-6}$, Andreas Görling ${ }^{2}$, Hans-Peter Steinrück ${ }^{1}$, Christian \\ Papp $^{1, *}$
}

1: Friedrich-Alexander-Universität Erlangen-Nürnberg (FAU), Lehrstuhl für Physikalische Chemie II, Egerlandstr. 3, 91058 Erlangen, Germany

2: Friedrich-Alexander-Universität Erlangen-Nürnberg (FAU), Lehrstuhl für Theoretische Chemie, Egerlandstr. 3, 91058 Erlangen, Germany

3: Friedrich-Alexander-Universität Erlangen-Nürnberg (FAU), Lehrstuhl für Mikro- und Nanostrukturforschung, Department Werkstoffwissenschaften, Cauerstr. 3, 91058 Erlangen, Germany

4: Department Interface Design, Helmholtz-Zentrum Berlin für Materialien und Energie GmbH (HZB), Albert-Einstein-Str. 15, 12489 Berlin, Germany

5: Energy Materials In-Situ Laboratory Berlin (EMIL), Helmholtz-Zentrum Berlin für Materialien und Energie GmbH (HZB), Albert-Einstein-Str. 15, 12489 Berlin, Germany

6: Helmholtz Institute Erlangen-Nürnberg für Renewable Energy (HI ERN), Albert-Einstein-Str. 15, Berlin, 12489, Germany

*: Christian.papp@fau.de

+: shared first authorship

\begin{abstract}
Supported catalytically active liquid metal solutions (SCALMS) have been receiving increasing attention recently. We investigated the oxidation behavior of macroscopic Rh-Ga alloy droplets and $\mathrm{Rh}-\mathrm{Ga}$ model catalyst nanoparticles supported on $\mathrm{SiO}_{2} / \mathrm{Si}(100)$ with low $\mathrm{Rh}$ content $(<2.5$ at \%) by $\mathrm{x}$-ray photoelectron spectroscopy in ultra-high vacuum and under near-ambient pressure conditions using different photon energies and also using transmission electron microscopy. The experiments are accompanied by computational studies on the Ga oxide/Rh-Ga interface and Rh$\mathrm{Ga}$ intermetallic compounds. For both Rh-Ga alloy droplets and Rh-Ga model catalyst nanoparticles, exposure to molecular oxygen leads to the formation of an oxide shell in which Rh is enriched. High-resolution transmission electron microscopy on the Rh-Ga nanoparticles confirms the formation of an approximately $4 \mathrm{~nm}$ thick gallium oxide film containing Rh. Based on ab-initio molecular dynamics and computational studies on the $\mathrm{Ga}_{2} \mathrm{O}_{3} / \mathrm{Ga}$ interface, it is concluded that $\mathrm{Rh}$ incorporation into the $\mathrm{Ga}_{2} \mathrm{O}_{3}$ film occurs by substituting octahedrally coordinated $\mathrm{Ga}$.
\end{abstract}




\section{Introduction}

Supported catalytically active liquid metal solutions (SCALMS) recently raised interest as highly active and selective dehydrogenation catalysts that outperform state-of-the-art industrial catalysts. Such SCALMS are oxide-supported particles of Ga-rich binary alloys, which contain small amounts (from 20 at.\% down to as low as 0.8 at.\%) of catalytically active metals such as Pd, Pt, or Rh. Knowledge about the exact surface composition is crucial to understand and further improve the catalytic behavior. At room temperature, the investigated alloy particles are biphasic, with a liquid transition-metal-depleted $G$ a phase and a solid transition-metal-enriched intermetallic phase (e.g.: $\mathrm{Ga}_{21} \mathrm{Rh}_{4}, \mathrm{Ga}_{16} \mathrm{Rh}_{3}$ in Rh-Ga model SCALMS particles).[1-5] Temperature-dependent $\mathrm{x}$ ray photoelectron spectroscopy (XPS) revealed that for macroscopic alloy droplets the crystallization of the intermetallic compounds (IMCS) occurs inside the bulk of the droplets, resulting in a transition-metal-depleted surface and near-surface region. Upon heating above the liquidus temperature, the IMCs dissolve in the liquid Ga phase, yielding a fully liquid alloy.[3-5] $A b$ initio molecular dynamics (AIMD) simulations showed that the near-surface region of liquid Pd-Ga, Pt-Ga, and Rh-Ga SCALMS systems has a layered structure along the surface normal: The first atomic layer is transition-metal-depleted, and the second layer is transition-metal-enriched with the expected bulk density approached over the next few layers. These results have been confirmed by angle-dependent XPS for the Pd-Ga system.[1, 3-5] Highly mobile, single transition metal atoms dynamically appearing as active centers at the transition-metal-depleted gas/liquid interface have been proposed as an explanation for the superior performance of SCALMS-based catalysts.[1, 3-8]

For the application of such catalysts, the formation of oxides upon exposure to ambient conditions and necessary activation processes to obtain the active liquid metal catalyst can play an important role. Using spatially resolved time-of-flight secondary ion mass spectrometry (TOFSIMS), Chabala studied the oxidation of pure liquid $\mathrm{Ga}$ at $27.7^{\circ} \mathrm{C}$, where liquid $\mathrm{Ga}$ is slightly supercooled.[9] Already at low oxygen partial pressures of $\approx 2 \times 10^{-9} \mathrm{mbar}$, they found the growth of low dimensional, stringy oxide structures, and at $\approx 7 \times 10^{-7} \mathrm{mbar}$, the Ga surface became covered with fractal-like oxide structures. No further increase of film thickness was observed at these pressures. Impurities are possible nucleation sites for the initial oxide formation on liquid surfaces, because typical nucleation sites on solid surfaces, like step edges or other defects, do not exist in these systems. Regan et al. investigated the oxidation of similar systems by X-ray reflectivity and grazing incidence X-ray scattering.[10-13] The presence of a quasi-Bragg reflection for the metallic liquid Ga phase shows the layering of the near-surface region along the surface normal. The loss of the quasi-Bragg reflection in the initial oxidation phase indicated an influence on the layering of liquid Ga near the Ga oxide/Ga interface. The obtained reflectivity of the oxidized surface was reproduced by both models, either assuming layered or non-layered liquid Ga below the Ga oxide/Ga interface. They found that both the liquid/oxide and the oxide/gas interface are 
atomically flat with below Ångstrom roughness. Furthermore, grazing incidence X-ray scattering suggested the formed oxide is most likely amorphous or only very poorly crystalline. Nevertheless, based on the determined reflectivity, they suggest an interface model for the liquid metal/oxide interface assuming that the formed oxide is similar to crystalline $\beta-\mathrm{Ga}_{2} \mathrm{O}_{3}$.

More recently, we performed a near-ambient-pressure (NAP) XPS study on the oxidation of liquid Ga and liquid Pt-Ga alloys with 0.7 and 1.8 at.\% Pt.[14] Similar to earlier results, we found that at oxygen pressures below $\approx 10^{-7}$ mbar the thickness of the oxide layer saturates at $(6.7 \pm 2.2) \AA \mathrm{Ga}_{2} \mathrm{O}_{3}$. For higher pressures, i.e. $10^{-3}$ and $1 \mathrm{mbar}$, we observed an initial rapid film growth, due to the formation of a closed, thin oxide layer, followed by slower film growth. This was interpreted as diffusion-limited film growth of a bulk oxide, due to the qualitative agreement with a square root like growth model. For the bulk oxide formation, no saturation was observed on the timescale of the conducted experiments. Notably, we found no differences between the film-growth kinetics of pure Ga and Pt-Ga alloys. The most striking result is the appearance and rapid initial increase of the $\mathrm{Pt} 4 \mathrm{f}$ signal during the oxidation, which indicates an oxidation-induced change in the Pt distribution, yielding an enrichment of Pt in the oxide layer.

In literature, the catalytic activity of Ga-based intermetallic compounds (with much higher active metal contents than in SCALMS) were investigated in some detail.[15] In particular, Pd-Ga based intermetallic compounds, which are selective catalysts for acetylene semihydration and are also considered for methanol steam reforming, were studied.[16] The phase diagram of the Pd-Ga system [17] provides a guideline to several other interesting IMCs. In contrast, the Rh-Ga phase diagram is only partially known, [18], and does not contain the IMC phases found in Rh-Ga SCALMS systems so far.[4] Haghofer et al. noticed the decomposition of $\mathrm{PdGa}$ and $\mathrm{Pd}_{2} \mathrm{Ga}$ in the presence of $\mathrm{O}_{2}$ under formation of $\mathrm{Ga}$ oxide and elemental $\mathrm{Pd}$ that accumulates at the surface.[19] Wowsnick et al. compared the oxidation of $\mathrm{Pd}_{2} \mathrm{Ga}$ in the form of a polished sample and milled $\mathrm{Pd}_{2} \mathrm{Ga}$ powder. For partial oxidation of the polished $\mathrm{Pd}_{2} \mathrm{Ga}$ specimen via trace $\mathrm{O}_{2}$ in the storage atmosphere, they observed a Pd surface enrichment by XPS. Upon oxidation of the milled $\mathrm{Pd}_{2} \mathrm{Ga}$ powder in air, a combined XPS and HR-TEM analysis revealed a non-uniform, amorphous Ga oxide layer, with metallic $\mathrm{Pd}$ and $\mathrm{Pd}_{2} \mathrm{Ga}$ nanoparticles of up to $5 \mathrm{~nm}$ size embedded in it. They also found that the reaction of milled $\mathrm{Pd}_{2} \mathrm{Ga}$ towards molecular oxygen depends on the oxidative treatment: when a previously reduced sample is carefully exposed to $\mathrm{O}_{2}$, Ga oxide forms on the surface but no $\mathrm{Pd}$ or $\mathrm{Pd}_{2} \mathrm{Ga}$ particle enrichment of the oxide film is observed. If, in contrast, the sample is abruptly exposed to air, elemental Pd particles get embedded in the formed oxide film.[20, 21] These differences show that oxidation of binary metal systems can vary depending on the chosen conditions.

For Pd-rich IMCs, electronic structure theory predicts a shift of the Pd $4 \mathrm{~d}$ valence states further below the Fermi level and consequently a charge transfer from Ga into previously unoccupied Pd $4 \mathrm{~d}$ states, which results in partial negative charging of the Pd centers.[22] Despite 
the fact that an increase in electron density typically results in XPS core level shifts to lower binding energy, positive shifts are reported for the Pd $3 \mathrm{~d}$ core level. Thereby the magnitude of the shift increases with the relative amount of Ga in the IMCs. [15, 23] Furthermore, the XPS line shape of the Pd $3 \mathrm{~d}$ core levels becomes more symmetric, which is attributed to a decreased probability for the creation of electron-hole pairs at the Fermi edge, due to the decreased density of unoccupied states in close proximity to the Fermi level.[20, 22, 23]

We present a combined XPS and density functional theory (DFT) study of the oxidation of macroscopic Ga-Rh droplets and microscopic Ga-Rh particles as model systems for Ga-Rh SCALMS with low Rh content: $\mathrm{Ga}_{125} \mathrm{Rh}_{1}$. The formation of $\mathrm{Ga}_{2} \mathrm{O}_{3}$ films wetting the surface of the macroscopic metallic alloys is investigated in situ using NAP XPS. The oxidation of macroscopic Rh$\mathrm{Ga}$ alloys leads to Rh surface enrichment. The lack of Rh 3d XPS signal attenuation implies Rh incorporation in the $\mathrm{Ga}_{2} \mathrm{O}_{3}$ film. As a complementary system, we also studied supported nanoalloy particles, which mimic the catalytic particles used in the application, with synchrotron-radiationbased XPS and scanning transmission electron microscopy (STEM). Furthermore, DFT-based AIMD simulations were conducted to construct different models for the oxide film formed on liquid Ga, which were subsequently used to calculate core level binding energy shifts to compare to experiments. The outcome is in good agreement with experiments, supporting the suggested assignment in the XPS spectra and showing that Rh is incorporated in octahedral sites in the oxide film.

\section{Experimental Section}

\section{Macroscopic Rh-Ga alloy preparation and laboratory-based NAP XPS measurements}

Gallium was purchased from Sigma-Aldrich/Alfa Aesar (99.99999 \%), Rhodium from Goodfellow (99.9\%). All examined samples were contained in tungsten crucibles purchased from Kurt Lesker $\mathrm{GmbH}$. The crucible was heated by resistive heating and its temperature was determined with a type $\mathrm{K}$ thermocouple spot-welded to its backside. The accuracy of the temperature reading is $\pm 2 \mathrm{~K}$, as estimated from the melting temperature of solid Ga.[14] The bimetallic Rh-Ga alloy with a molar ratio of $\mathrm{Ga} / \mathrm{Rh}=125$ (hereafter, $\mathrm{Ga}_{125} \mathrm{Rh}_{1}$ ) was prepared by introducing corresponding weights of liquid $\mathrm{Ga}$ and a Rh wire into a tungsten crucible in air. After introduction to the ultrahigh vacuum (UHV) system, the mixture was heated to $850 \mathrm{~K}$ to facilitate alloying and to desorb native oxides. During heating, the dissolution of the Rh wire into the Ga was observed visually. Furthermore, the sample was cleaned by $\mathrm{Ar}^{+}$sputtering ( $1 \mathrm{keV}, 10^{-5} \mathrm{mbar} \mathrm{Ar}$ ). Quantitative XPS of the cleaned sample at $810 \mathrm{~K}$ suggests a composition of 0.71 at.\% Rh in Ga which, within the experimental uncertainty, matches the 0.79 at.\% expected based on weigh in. Oxygen was purchased from Linde ( $99.9997 \%)$. The oxygen pressures were adjusted by background dosing. After each oxidation cycle, the formed gallium oxide was desorbed by heating the alloy to $850 \mathrm{~K}$ in 
UHV. To verily the cleanliness of the surface $O 1 s, C 1 s$ and Ga $2 p$ regions were checked before the next oxidation experiment. This cleaning procedure was found to be sufficient, no sputtering between the measurements was necessary to prepare an oxygen and carbon free surface.

For the studies on the macroscopic Rh-Ga alloys, we used a lab-based NAP-XPS setup with a $\mathrm{Mg} / \mathrm{Al} \mathrm{K}_{\alpha}$ twin anode (SPECS XR-50 HP) and a modified hemispherical electron energy analyzer (OMICRON EA 125). The X-ray source is non-monochromatized and the sample irradiated at an incidence angle of $65^{\circ}$ with respect to the surface normal. The data shown in this publication is measured using $\mathrm{Al} \mathrm{K}_{\alpha}$ radiation. All spectra were collected in normal emission. The instrumental broadening (FWHM of the Gaussian) of the setup was determined to $1.25 \mathrm{eV}$. The setup operates at a base pressure of $10^{-9} \mathrm{mbar}$; for details see Ref.[24].

For data evaluation and quantitative analysis of the recorded data, we used the Casa XPS software package. To compensate for fields induced by resistive heating, we aligned the binding energy scales of measurements conducted above room temperature to the metallic $\mathrm{Ga} 2 \mathrm{p}$ signal. Based on the scatter of the energy position of the valence band edge, we estimate an experimental uncertainty of $\pm 0.1 \mathrm{eV}$ for the given absolute binding energies. Symmetric signals were fitted using Voigt functions, and for metallic signals an asymmetric Voigt-like line shape (denoted as LF in Casa XPS) was applied. This lineshape was found to reproduce the metallic signals of a Ga reference best.

The oxide film thicknesses $D$ on the macroscopic Ga droplet were evaluated based on a layer model by Jeurgens et al.[25]. In this model the thickness $D$ is obtained from the intensity ratio of the oxidic component $l_{i, o x}$ and the metallic component $l_{i, \text { met }}$ of the Ga signals:

$$
D=\lambda_{i, G a 203} \ln \left(\frac{I_{i, O x} \lambda_{i, G a} N_{G a}}{I_{i, m e t} \lambda_{i, G a 2 O 3} N_{G a 2 O 3}}+1\right)
$$

The subscript $i$ denotes the core level (Ga $3 d$ or Ga 2p), $\lambda$ is the inelastic mean free path (IMFP) in either gallium $\left(\lambda_{i, G a}\right)$ or gallium oxide $\left(\lambda_{i, G a 2 O 3}\right)$, and $N_{G a}$ and $N_{G a 2 O 3}$ are the Ga densities in metallic $\mathrm{Ga}$ and in $\beta-\mathrm{Ga}_{2} \mathrm{O}_{3}$, respectively. The influence of the $\mathrm{Rh}$ atoms in $\mathrm{Ga}$ and $\mathrm{Ga}_{2} \mathrm{O}_{3}$ on the IMFP values was neglected. IMFP values were calculated according to the non-relativistic TPP-2M algorithm.[26] The film thicknesses were independently evaluated from both the Ga $3 d$ and the Ga $2 p$ intensities. The obtained values agreed within the expected experimental error, and the values presented in the results section are averages of the values obtained for the two core levels.

\section{Particle preparation for TEM studies and synchrotron-radiation-based XPS measurements}


The nanoalloy particles used for the electron microscopy studies and the synchrotron-radiationbased XPS measurements were prepared using physical vapor deposition (PVD). For the microscopy studies, a Focus EMF3-T evaporator and as substrate $\sim 30 \mathrm{~nm}$ thick $\mathrm{SiN}_{\mathrm{x}}$ or $\mathrm{SiO}_{\mathrm{x}}$ supporting membranes were used. For the synchrotron studies a SPECS EBE-4 four-pocket electron beam evaporator were used. In both cases, the deposition rate was determined by a quartz crystal microbalance. For the synchrotron studies, a natively oxidized $\left(\mathrm{SiO}_{\mathrm{x}}\right) / \mathrm{Si}$ substrate was introduced in UHV as received. Subsequently, Rh and Ga were deposited at room temperature; formation of intermetallic phase as precipitation in the Ga-rich amorphous / liquid alloy droplets without heating the substrate during deposition and without post deposition heat treatment was observed by TEM. The pressure during deposition was $\approx 9 \cdot 10^{-9} \mathrm{mbar}$. Rh was evaporated first using a $1 \mathrm{~mm}$ thick wire from Sigma-Aldrich with an evaporation rate of $0.01 \AA / \mathrm{s}$ and a total nominal thickness of $2.2 \AA$. Ga was evaporated subsequently from an $\mathrm{Al}_{2} \mathrm{O}_{3}$ crucible with an evaporation rate of $0.06 \AA$ \&s and a total nominal thickness of $123 \AA$. After deposition, the samples were transferred to the measurement position without breaking UHV conditions.

The XPS measurements were conducted at the SISSY I endstation permanently installed the dual color beamline in the Energy Materials In-situ Laboratory Berlin (EMIL) at HZB. SISSY I is equipped with a Scienta EW 4000 electron energy analyzer and is operated at UHV conditions (base pressure $<2 \cdot 10^{-9} \mathrm{mbar}$ ). Photon energies of 449 and $1045 \mathrm{eV}$ provided by the plane grating monochromator and APPLE II-type UE 48 undulator of the soft X-ray branch of the EMIL beamline. For all core level scans, the pass energy was $50 \mathrm{eV}$ and the energy step width $0.1 \mathrm{eV}$. The binding energy was calibrated using a clean Au foil as reference, setting the $A u 4 f_{7 / 2}$ core level line to 84.00 $\mathrm{eV}$, or setting the center of the measured Fermi level to $0.00 \mathrm{eV}$. The total energy resolution was determined from the width of the Fermi level of a clean polycrystalline Au sample to be $0.27 \mathrm{eV}$ at $449 \mathrm{eV}$ and $0.58 \mathrm{eV}$ at $1045 \mathrm{eV}$.

(Scanning) transmission electron microscopy (S/TEM) studies were performed on a Titan ${ }^{3}$ Themis microscope operating at either 200 or $300 \mathrm{kV}$. The TEM is equipped with Cs-correctors both at the illumination and imaging side, along with a Super-X energy dispersive $X$-ray (EDX) detector and a GIF Quantum ERS (spectrometer). The Super-X detector comprises four silicon drift detectors (SDD) symmetrically placed around the optical axis, close to the sample area. All four signals are combined into one spectrum to improve the collection efficiency in spectrum imaging (SI) acquisition. In the STEM imaging and SI experiments, the probe-forming semi-angle was set to a value of $15.7 \mathrm{mrad}$. A probe current of 200-500 pA, dwell time of 30-50 $\mu$ s and typically 100-200 frames summation were applied. High-angle annular dark-field (HAADF), ADF, annular bright-field $(A B F)$, and BF STEM images were simultaneously acquired along with the EDX signals.

\section{Computational Details}


All periodic DFT calculations were performed with the Vienna Ab Initio Simulation Package (VASP), which uses a plane wave basis set. The projector augmented wave (PAW) method was chosen to represent the atomic cores,[27-29] and the PBE functional to describe the exchange-correlation part.[30] To check possible errors arising from the insufficient description of some properties by the PBE functional, the HSEO6 hybrid functional with the amount of exact exchange set to 0.35 to reproduce the experimental band gap and lattice constants was chosen for some calculations.[31] A dipole correction was applied in the direction perpendicular to the surface. A cutoff of $415 \mathrm{eV}$ was used for the plane wave basis set. Calculations for pure $\mathrm{Ga}$ or gallium-oxide $\left(\mathrm{Ga}_{2} \mathrm{O}_{3}\right)$ systems were non-spin-polarized, but as soon as the $\mathrm{Ga}_{2} \mathrm{O}_{3}$ systems were doped with Rh, spin-polarized calculations were performed.

We carried out AIMD simulations in combination with geometry optimizations to generate different liquid Ga / Ga-oxide systems, which are described below. The equations of motion in the AIMD simulations were integrated using a Verlet algorithm with a timestep of $2 \mathrm{fs}$ for the $\mathrm{Ga}_{2} \mathrm{O}_{3}$ systems. To simulate a canonical ensemble a Nosé-Hoover thermostat was used with temperatures as described in the computational models section (see SI) and Fermi smearing corresponding to the temperature conditions.[32, 33] AIMD simulations were carried out at the $\Gamma$ point, while geometry optimizations and calculation of core level energies were carried out with an increased $2 \times 2 \times 1 \mathrm{k}$-point mesh. The convergence criterion for the wave function was set to $10^{-6}$ $\mathrm{eV}$ and $10^{-7} \mathrm{eV}$ in AIMD simulations and geometry optimizations, respectively. Structure relaxations were carried out until the maximal Cartesian force component was lower than 0.05 $\mathrm{eV} / \AA ̊$. For pure metallic Rh and Ga-Rh systems a Methfessel-Paxton smearing of first order was chosen with a width of $0.2 \mathrm{eV}$.[34]

For comparison with experimental findings, we computed core level binding energies $\varepsilon_{\mathrm{CL}}$ in the final state approximation by removing one core electron (Rh $3 d$ and $\mathrm{Ga} 2 \mathrm{p} / \mathrm{Ga} 3 \mathrm{~d}$, respectively) of the corresponding atom and instead placing it at the Fermi level. Subsequently, the electronic system is relaxed to describe the response of the valence electrons to the generated core hole. The drawback of the PAW method is that the core electrons are frozen and therefore effects arising from the core electrons are not taken into account. For that reason, absolute core level binding energies cannot be compared to experiments. However, if binding energie shifts are considered, that is, differences between different atoms of the same species, the errors should nearly cancel which makes a comparison to shifts in experimental data possible. These core level shifts (CLS) are calculated as: $\mathrm{E}(\mathrm{CLS})=\varepsilon_{\mathrm{cl}}-\varepsilon_{\mathrm{cl}}$, ref, where $\varepsilon_{\mathrm{cl}}$ is the core level binding energy of the investigated atom and $\varepsilon_{\mathrm{cl}}$, ref is that of the reference atom. Additionally, the core level binding energies are referenced to the Fermi level $\varepsilon_{\mathrm{F}}$ by $\varepsilon_{\mathrm{CL}}=-\left(\varepsilon_{\mathrm{C}}-\varepsilon_{\mathrm{F}}\right)$ with $\varepsilon_{\mathrm{C}}$ as the absolute core level energy. We also tested a different approach where the excited electron is removed completely instead of adding it to the Fermi level, which yields very similar results. Here, a further approximation is included by removing the electron from the system and therefore charging the 
unit cell, which is unphysical due to the periodic boundary conditions. This is prevented by using a negative background charge smeared across the whole unit cell.

To estimate the accuracy of our results, we calculated the core level shifts between metallic $\mathrm{Ga}$ and $\mathrm{Ga}_{2} \mathrm{O}_{3}$ for $\mathrm{Ga} 2 \mathrm{p}$ as well as $\mathrm{Ga} 3 \mathrm{~d}$ electrons. For both types of excitations, the core level shift is well known to be around $+2.0 \mathrm{eV}$, i.e. $2 \mathrm{eV}$ towards higher binding energy, for the oxide compared to the metallic Ga. We chose different PAWs, either including the $3 \mathrm{~d}$ electrons in the core (for $\mathrm{Ga} 3 \mathrm{~d}$ ) or treating them as valence (for Ga $2 \mathrm{p}$ ). For Rh, the $3 \mathrm{~d}$ electrons were excited from the core and $5 \mathrm{~s}$ as well as $4 \mathrm{~d}$ electrons were treated explicitly as valence electrons.

Charges were calculated in the Bader approach from the all-electron density.[35, 36] The density of states (DOS) was calculated using increased k-point meshes. 


\section{Results and Discussion}

\section{Oxidation of macroscopic alloys}

The prepared samples were exposed to molecular oxygen at pressures of $1 \mathrm{mbar}$ (equivalent to a dose of $\left.4.9 \times \cdot 10^{9} \mathrm{~L} \mathrm{O}_{2}\right), 10^{-2} \mathrm{mbar}\left(1.7 \times 10^{8} \mathrm{~L} \mathrm{O}_{2}\right), 10^{-3} \mathrm{mbar}\left(1.4 \times 10^{7} \mathrm{~L} \mathrm{O}_{2}\right), 10^{-5} \mathrm{mbar}\left(1.1 \times \cdot 10^{5} \mathrm{~L}\right.$ $\mathrm{O}_{2}$ ) and $10^{-7} \mathrm{mbar}$ ( $700 \mathrm{~L} \mathrm{O}_{2}$, not shown) at $550 \mathrm{~K}$ for times between 108 and 376 minutes. In all cases, the formation of $\mathrm{Ga}_{2} \mathrm{O}_{3}$ was observed. Figure 1 displays the spectra of the $\mathrm{Ga} 3 \mathrm{~d}, \mathrm{Ga} 2 \mathrm{p}_{3 / 2}$, $\mathrm{Rh} 3 \mathrm{~d}$, and $\mathrm{O} 1 \mathrm{~s}$ regions after the $\mathrm{Ga}_{2} \mathrm{O}_{3}$ films were grown by exposing the $\mathrm{Rh}_{1} \mathrm{Ga}_{125}$ alloy to up to 4.9. $\times 10^{9} \mathrm{~L} \mathrm{O}_{2}$ at $550 \mathrm{~K}$. In addition, the $\mathrm{Rh}_{1} \mathrm{Ga}_{125}$ alloy was exposed to $\mathrm{O}_{2}$ at $305 \mathrm{~K}$ (data shown in the $\mathrm{SI})$, at pressures of $3 \times 10^{-7} \mathrm{mbar}\left(4.4 \times \cdot 10^{3} \mathrm{~L} \mathrm{O}_{2}\right), 3 \times 10^{-5} \mathrm{mbar}\left(4.4 \times \cdot 10^{5} \mathrm{~L} \mathrm{O}_{2}\right)$ and $1 \mathrm{mbar}(8.9$ $x \cdot 10^{9} \mathrm{~L} \mathrm{O}_{2}$ ), to evaluate the influence of temperature on the oxide formation (see below). 

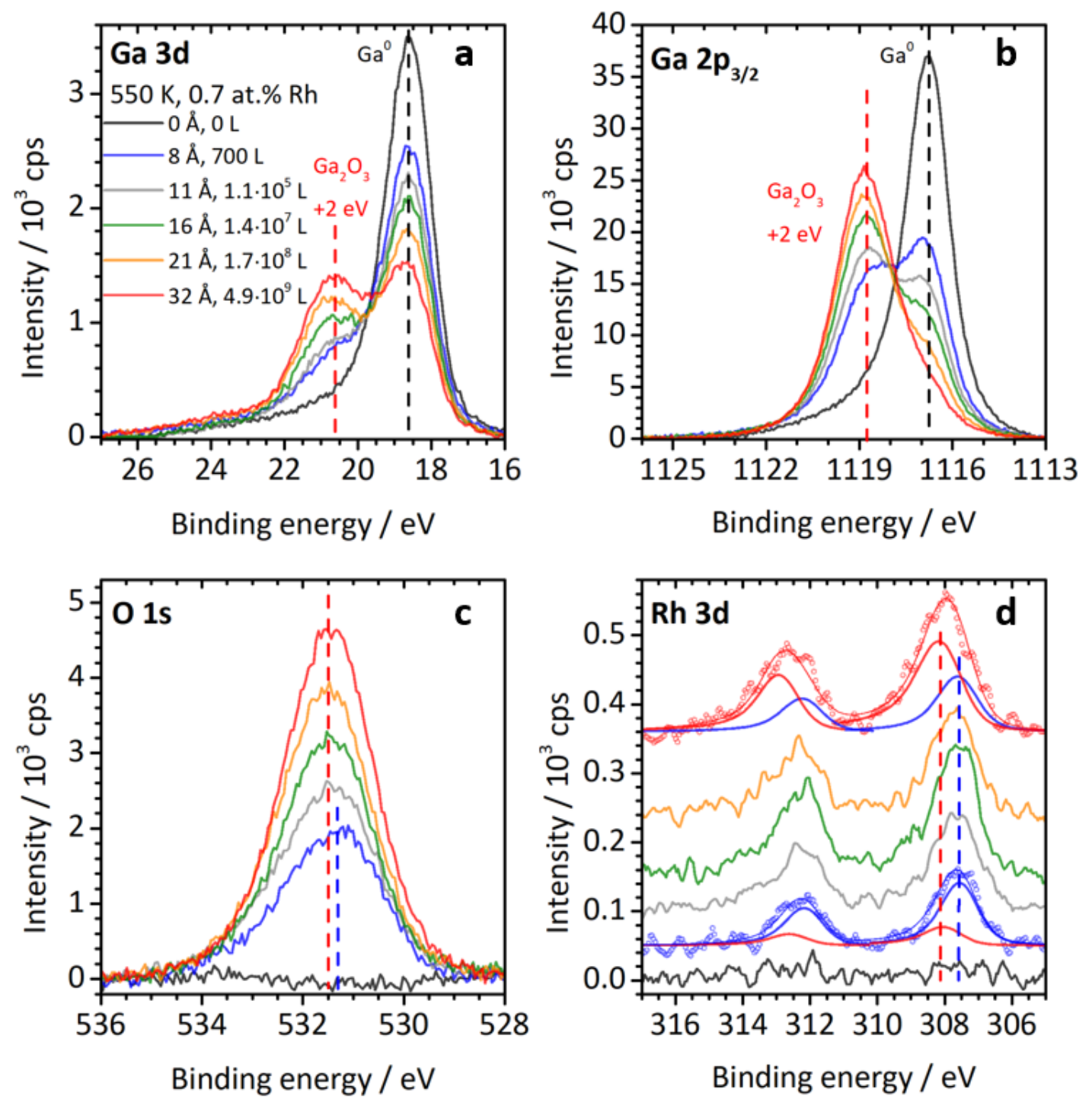

Figure 1:XPS (Al $\left.K_{\alpha}, h v=1486.58 \mathrm{eV}\right)$ results of the oxidation of a macroscopic Rh/Ga alloy droplet with a molar ratio of $G a / R h=125$ (i.e., 0.7 at\% Rh) at $550 \mathrm{~K}$. (a) and (b) show the $\mathrm{Ga} 3 d$ and $\mathrm{Ga} 2 p_{3 / 2}$ core levels, after different oxygen doses. The $\mathrm{Ga}_{2} \mathrm{O}_{3}$ film thicknesses in Å given in (a) were derived from the ratio of the oxidic to the metallic component of the Ga $2 p$ signal as is outlined in the experimental section. Panel (c) shows the corresponding $O 1 s$ region, and (d) the $R h 3 d$ region. The latter also show the two fits for the lowest and highest oxygen doses.

All spectra displayed in Figure 1 were recorded in UHV after exposure to oxygen. Notably, spectra recorded in situ during oxidation show the same binding energies (see SI, Figure S1). The Ga 3d signal in Figure 1a consists of two spin-orbit split components that are not resolved in the lab experiment. The metallic gallium, $\mathrm{Ga}^{0}$, peak at $18.6 \mathrm{eV}$ is indicated by a black dashed line, and the peak position of the newly formed oxide, $\mathrm{Ga}_{2} \mathrm{O}_{3}$, at $20.6 \mathrm{eV}$ by a red dashed line. For the $\mathrm{Ga} 2 \mathrm{p}$ signal in Figure $1 \mathrm{~b}$ only the $2 \mathrm{p}_{3 / 2}$ component is shown, with the $\mathrm{Ga}^{0}$ peak at 1116.7 and the $\mathrm{Ga}_{2} \mathrm{O}_{3}$ peak at $1118.7 \mathrm{eV}$. The observed binding energy shift of $+2.0 \pm 0.15 \mathrm{eV}$ between the oxidic and 
metallic Ga $3 d$ and Ga 2 $p_{3 / 2}$ components is in agreement with values in literature.[37-40] Note that for oxidation at $305 \mathrm{~K}$ and low pressures $\left(3 \times 10^{-7}\right.$ and $\left.3 \times 10^{-5} \mathrm{mbar}\right)$, the oxide components appear at slightly lower binding energies of 20.2 and $1118.3 \mathrm{eV}$ in the Ga $3 \mathrm{~d}$ and $\mathrm{Ga} 2 \mathrm{p}_{3 / 2}$ regions, respectively. With increasing exposure to oxygen, the spectra show in all experiments an increase in the oxide signals and a decrease (attenuation) in the metallic signals. The attenuation of the metallic $\mathrm{Ga}^{0} 2 \mathrm{p}_{3 / 2}$ peak intensity (see Fig. 1 b) due to the oxidation process is much stronger than the one observed in the $\mathrm{Ga}^{0} 3 \mathrm{~d}$ signal, which, due to the different kinetic energies of the emitted photoelectrons ( $\mathrm{E}_{\mathrm{Kin}}=\approx 370 \mathrm{vs} . \approx 1460 \mathrm{eV}$ ), is a clear indication that the signal is being attenuated by an overlying oxide layer. The stronger attenuation of the surface-sensitive signal suggests that the oxide film mainly forms at the alloy/gas interface and that it completely covers (wets) the surface of the liquid alloy.

In parallel, in the $\mathrm{O} 1 \mathrm{~s}$ region (Figure $1 \mathrm{c}$ ) a signal is observed at $531.5 \mathrm{eV}$ (red dashed line) that increases with increasing exposure to $\mathrm{O}_{2}$. This signal is assigned to oxygen in $\mathrm{Ga}_{2} \mathrm{O}_{3}$. For a very thin oxide film ( $8 \AA$, blue line) a slightly lower value of $531.2 \mathrm{eV}$ is found. This binding energy is similar to the early stage of Pt-Ga alloy oxidation, where only a $2 \mathrm{D}$ oxide film covers the surface.[14] By comparing the integrated peak areas of the oxide components in the $\mathrm{Ga} 3 \mathrm{~d}$ and $\mathrm{Ga} 2 \mathrm{p}$ regions to that of the $\mathrm{O} 1 \mathrm{~s}$ signal, the $\mathrm{Ga} / \mathrm{O}$ stoichiometry of the grown oxide films was determined to be 0.75 , with a standard deviation of \pm 0.10 (for details see SI, Table S1). Within the accuracy of the experiment, this is reasonably close to the value of 0.67 expected for $\mathrm{Ga}_{2} \mathrm{O}_{3}$, the commonly found stoichiometry of gallium oxide. This stoichiometry was also used in the theoretical calculations discussed below.

Most remarkable is the development in the $\mathrm{Rh} 3 \mathrm{~d}$ region shown in Figure $1 \mathrm{~d}$ during oxygen exposure. The initial spectrum of the metallic alloy ( $0 \AA$, black) does not show any significant Rh $3 d$ signal intensity. This is in line with expectations: at $550 \mathrm{~K}$, the Rh-Ga system is still biphasic, having not yet reached the liquidus temperature. Most Rh is therefore bound in solid intermetallic compounds within the bulk of the droplets, and thus is not detectable by XPS. After oxygen exposure, the spin-orbit-split $R h 3 d_{5 / 2} / 3 d_{3 / 2}$ doublet becomes visible in the $\mathrm{Rh} 3 \mathrm{~d}$ region. The binding energy of the $R h 3 d_{5 / 2}$ peak is initially 307.6 and increases to $308.0 \mathrm{eV}$ with increasing exposure to $\mathrm{O}_{2}$. Note that an oxidation-induced enrichment of the transition metal at the surface was also observed for Pt-Ga alloys.[14] We analyzed the data by introducing two spin-orbit signals at fixed binding energies and the expected ratio of 6:4, positioned at 307.6 and $308.0 \mathrm{eV}$ for the $\mathrm{Rh} 3 \mathrm{~d}_{5 / 2}$ and 312.4 and $312.8 \mathrm{eV}$ for the $\mathrm{Rh} 3 \mathrm{~d}_{3 / 2}$ contributions, see Fig. $1 \mathrm{~d}$. For the observations described within the following, any relative change in binding energy or intensity observed for the $R h 3 d_{5 / 2}$ components is in the same way observed for the Rh $3 d_{3 / 2}$ components. Thus, we will limit our discussion to only the $\mathrm{Rh} 3 \mathrm{~d}_{5 / 2}$ components. Despite the low signal-to-noise ratio it is clear that the observed binding energies of the two Rh $3 \mathrm{~d}_{5 / 2}$ components are shifted by +0.2 and $+0.6 \mathrm{eV}$ to higher binding energy compared to the measured signal of a $\mathrm{Rh}(111)$ single crystal. Note that the 
observed binding energy do not match typical values reported for bulk $\mathrm{Rh}_{2} \mathrm{O}_{3}$, which are reported to be between $+0.9 \mathrm{eV}$ [41] and $+1.6 \mathrm{eV}$ [42] with respect to the binding energy of the $\mathrm{Rh}(111)$ single crystal.

The quantitative analysis of the in situ recorded $\mathrm{Ga} 3 \mathrm{~d}, \mathrm{Ga} 2 \mathrm{p}_{3 / 2}, \mathrm{O} 1 \mathrm{~s}$, and Rh $3 \mathrm{~d}$ intensities during oxidation under $10^{-2}$ mbar $\mathrm{O}_{2}$ pressure are shown in Figure 2a; for the corresponding spectra see Figure S1 in the SI. The decrease of the metallic components (open circles) and the increase of the oxide components (full circles) are clearly visible. The formation of the oxide layer monitored in situ can be described as a two-stage process. In the early phase, rapid film growth occurs for 20 to $60 \mathrm{~min}$ (depending on the pressure, i.e., the $\mathrm{O}_{2}$ dose), which is followed by a slow, linear - or possibly square root-like - behavior. No saturation of the film growth was observed on the timescale of the experiments. A likely explanation for the two-stage behavior is the quick formation of a thin 2D oxide film, which is followed by slow, mass-transport-limited 3D growth. A similar behavior was also observed for Pt-Ga alloys.[9, 13, 14]

In Figure $2 \mathrm{~b}$, the intensity development of the $\mathrm{O} 1 \mathrm{~s}$ and the Rh $3 \mathrm{~d}$ signals are compared. For both signals, a rapid initial increase is observed. For the $O 1$ s signal, this is followed by an almost linear - or possibly square root-like - increase, similar to that observed for the oxidic components of the $\mathrm{Ga}$ core levels. After the rapid initial increase, the Rh $3 \mathrm{~d}$ signal stays virtually constant, only minor increases are observed in some cases. This behavior indicates that $\mathrm{Rh}$ is incorporated into the $\mathrm{Ga}_{2} \mathrm{O}_{3}$ film at the early stages of $\mathrm{Ga}$ oxidation and that it remains in the near-surface region during the later stages of growth. One explanation could be further oxide growth at the interface of the formed oxide film with the liquid phase underneath by oxygen diffusion through the oxide without further inclusion of Rh atoms. Figure $2 c$ shows the Rh content in $G a\left(x_{R h}=\left(N_{R h} /\left(N_{R h}+N_{G a}\right)\right) * 100 \%\right)$ as function of final $\mathrm{Ga}_{2} \mathrm{O}_{3}$ film thickness. Both $\mathrm{Rh}$ content and film thickness shown here were determined based on the UHV measurements after oxidation at different pressures. Oxidations at $550 \mathrm{~K}$ are color-coded in black, oxidations at $305 \mathrm{~K}$ in blue. Note that the dashed line in $2 \mathrm{c}$ is a guide to the eye, indicating an overall trend of the measurements. Overall, the obtained Rh content is a function of pressure, that is, with increasing pressure a higher Rh content is found. Decreased temperature $(305 \mathrm{~K})$ results in the same effect within the error bar of the experiment, spectra are given in the SI.

To pinpoint the origin of the observed Rh surface enrichment, we analyzed the binding energy shifts in the Rh $3 \mathrm{~d}$ signals observed during the experiments more closely. For this purpose, Figure 3 compares Rh $3 d$ and $\mathrm{Ga} 3 \mathrm{~d}$ spectra of pure $\mathrm{Rh}(\mathrm{Rh}(111))$ and pure liquid $\mathrm{Ga}$ as references (black), a fully liquid Rh-Ga alloy with single Rh atoms in a Ga matrix (orange), a Rh-Ga intermetallic compound, likely $\mathrm{Ga}_{21} \mathrm{Rh}_{4}$ or $\mathrm{Ga}_{16} \mathrm{Rh}_{3}$, (blue), with two $\mathrm{Rh}$-Ga alloys after oxidation; one was oxidized more gently at $10^{-2} \mathrm{mbar}$ (green), and one more rapidly at $1 \mathrm{mbar}$ (red). For our oxidized Rh-Ga alloys, we find that the Rh $3 d_{5 / 2}$ binding energy is shifted between $+0.2(307.6 \mathrm{eV})$ and +0.6 
$\mathrm{eV}(308.0 \mathrm{eV})$ to relative to our $\mathrm{Rh}(111)$ reference $(307.4 \mathrm{eV})$. We interpret this to the existence of two different components (see fits of the red and green spectrum). In all experiments, it was possible to reproduce the $\mathrm{Rh} 3 \mathrm{~d}$ signal envelope by a superposition of these two components. The comparison of these binding energies to the value of $307.4 \mathrm{eV}$ for $\mathrm{Rh}(111)$ and $307.1 \mathrm{eV}$ for a fully liquid Rh-Ga system excludes the formation of a pure metallic Rh phase or a Rh enrichment in the liquid Rh-Ga alloy below the formed oxide film as possible contributions. The binding energy of $307.6 \mathrm{eV}$ matches well with our observations for a Rh-Ga intermetallic compound that crystallized at the Ga/vacuum interface. For the non-oxidized Rh-Ga alloy, it is rather rare for the IMCs to crystallize at the vacuum interface; nevertheless, this was observed occasionally during the conducted experiments. For low oxygen pressures, such Rh surface enrichment by formation of $\mathrm{Rh}-\mathrm{Ga}$ intermetallic compounds at, or incorporated into the rather thin $\mathrm{Ga}_{2} \mathrm{O}_{3}$ film seems reasonable, since the growing interface can be expected to provide additional heterogeneous nucleation sites.

The $\mathrm{Rh} 3 \mathrm{~d}_{5 / 2}$ component at $308.0 \mathrm{eV}$ is assigned to $\mathrm{Rh}$ incorporated in $\mathrm{Ga}_{2} \mathrm{O}_{3}$ by substituting $\mathrm{Ga}$ atoms in octahedral oxygen coordination based on the computational study of the $\mathrm{Ga}_{2} \mathrm{O}_{3} / \mathrm{Ga}-\mathrm{Rh}$ interface discussed below. Note that when comparing the $\mathrm{Rh} 3 \mathrm{~d}$ line shape of $\mathrm{Rh}(111)$ to that of $\mathrm{Rh}$ in the liquid or intermetallic alloy, a slight loss in asymmetry is found. This effect will be discussed in more details for the high-resolution synchrotron XPS data below. 

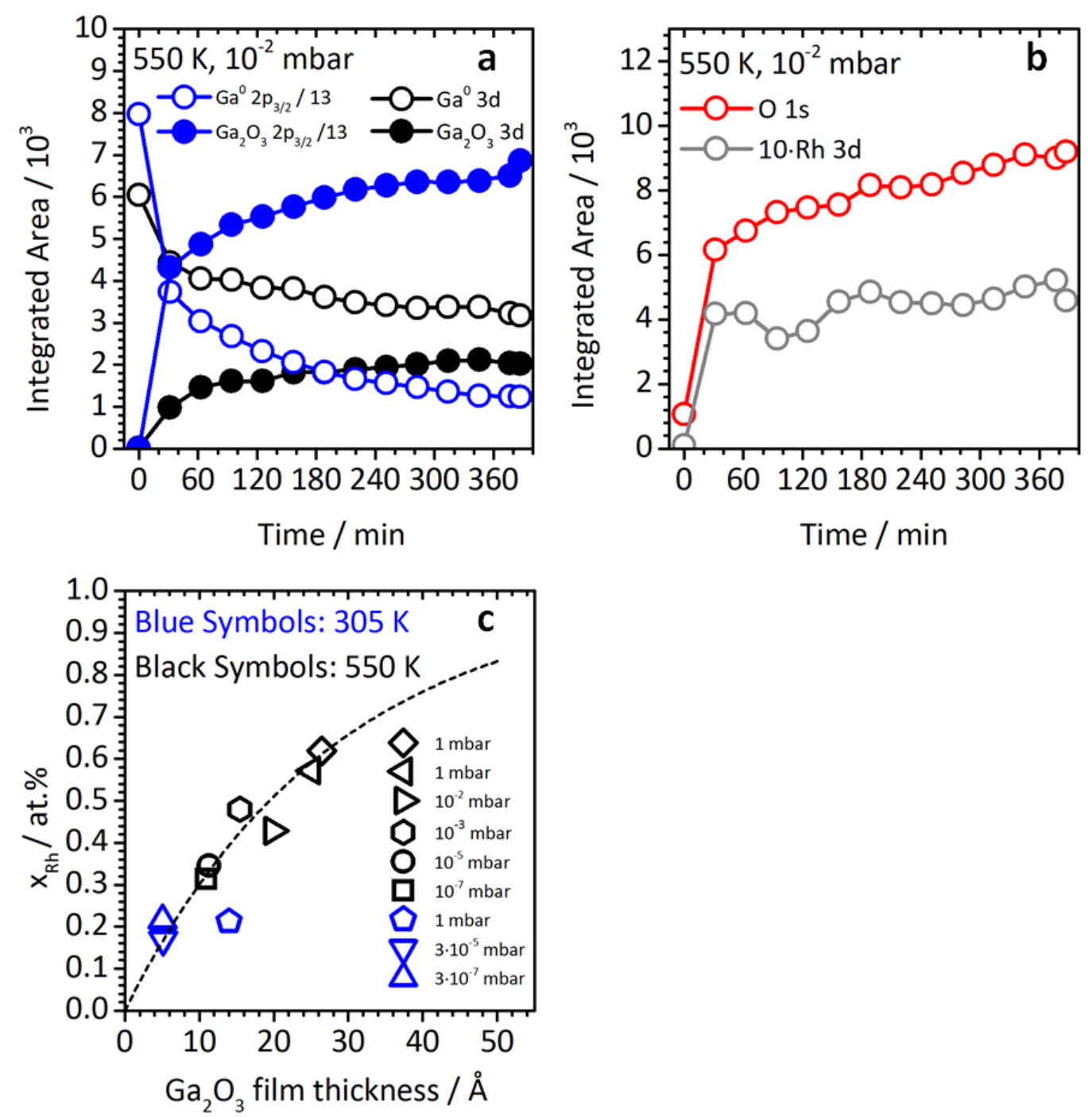

Figure 2 (a) $G a 3 d$ and $G a 2 p_{3 / 2}$ in situ data of the oxidation of a macroscopic $R h / G a$ alloy droplet with a molar ratio of $\mathrm{Ga} / \mathrm{Rh}=125$ in the presence of $1 \times \cdot 10^{-2}$ mbar oxygen at $550 \mathrm{~K}$. (b) Respective $O$ 1s and Rh $3 d$ intensities. (c) Rh content measured in UHV after oxidation in different pressures at 305 and $550 \mathrm{~K}$ as a function of the obtained $\mathrm{Ga}_{2} \mathrm{O}_{3}$ film thicknesses.

To summarize our studies on a macroscopic $\mathrm{Rh} / \mathrm{Ga}$ alloy droplet with a molar ratio of $\mathrm{Ga} / \mathrm{Rh}=$ 125 , we conclude that oxidation under the described conditions results in a Rh surface enrichment ascribed to the formation of Rh-Ga intermetallic compounds, and the incorporation of Rh into the forming $\mathrm{Ga}_{2} \mathrm{O}_{3}$ film by substituting $\mathrm{Ga}$. While oxidation-induced $\mathrm{Rh}$ surface enrichment is observed for all investigated pressures, the nature of the enrichment seems to depend on the chosen pressure (i.e., $\mathrm{O}_{2}$ dose). It is of high relevance to check whether these findings are transferable from pure model systems to more realistic particle systems. 

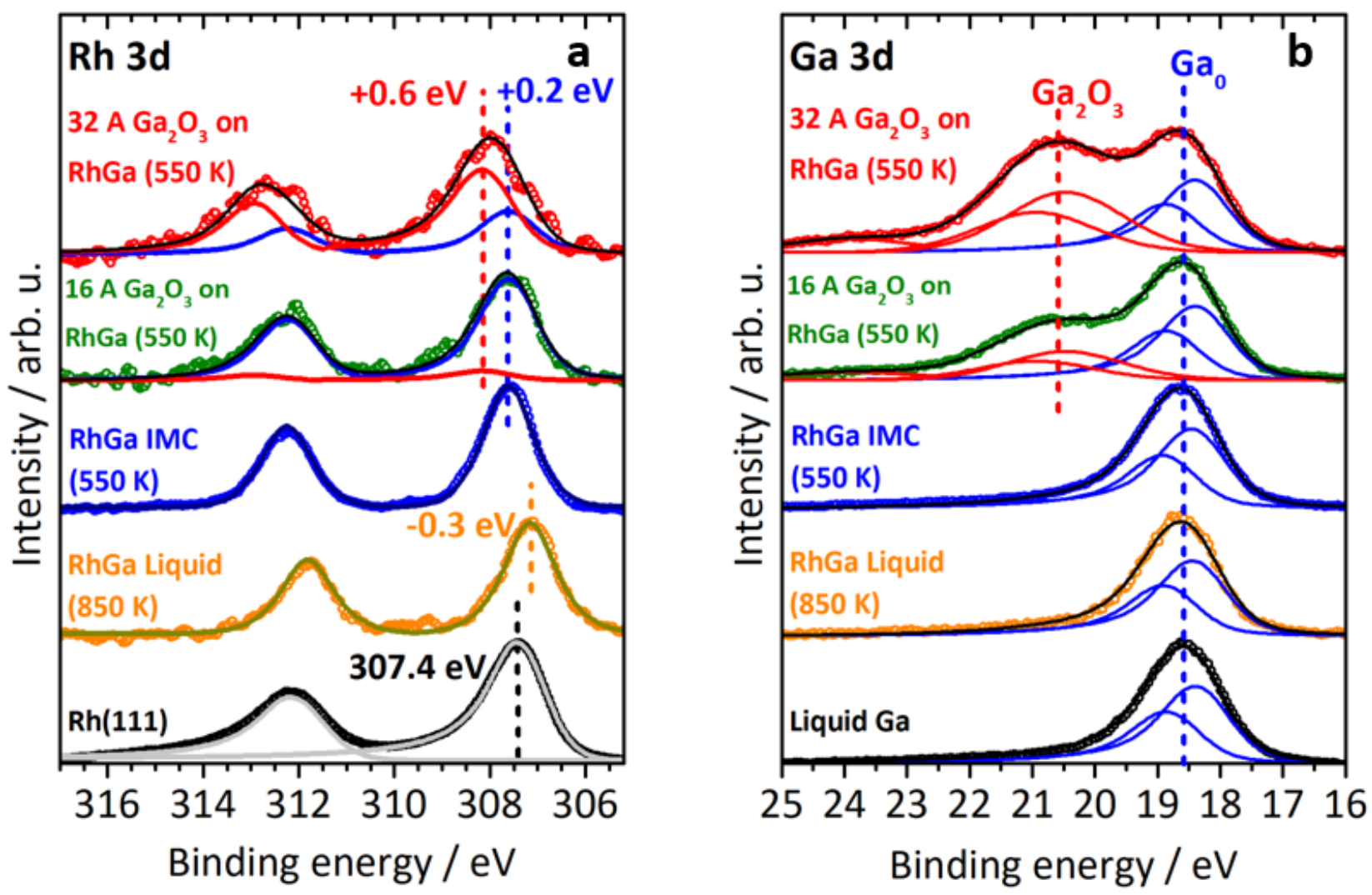

Figure 3: (a) Rh 3d and (b) Ga 3d spectra taken of a $R h(111)$ single crystal or liquid Ga (black), fully liquid $R h$-Ga at $850 \mathrm{~K}(G a / R h=125$; orange), a Rh-Ga intermetallic compound crystallized at the vacuum/Ga interface (see text; blue) and two spectra (green and red) recorded in in UHV after oxidation of a macroscopic Rh/Ga alloy droplet In the Ga $3 d$ region the growth of an additional peak with higher binding energy is observed, indicating oxide growth. The $\mathrm{Rh} 3 d$ peaks show characteristic shifts.

\section{Oxidation of supported model SCALMs}

As a model system for complementary studies using STEM and spatially resolved EDX (i.e., STEMEDX) mapping, we prepared Rh-Ga nanoparticles by physical vapor deposition onto thin $\mathrm{SiN}_{\mathrm{x}}, \mathrm{SiO}_{\mathrm{x}}$ or carbon membranes of $\approx 30 \mathrm{~nm}$ thickness, which are suitable for TEM studies. We found that the phase composition and morphology of the particles show no dependence on the choice of membranes. These deposited nanoparticles are expected to closely resemble the real catalyst. 


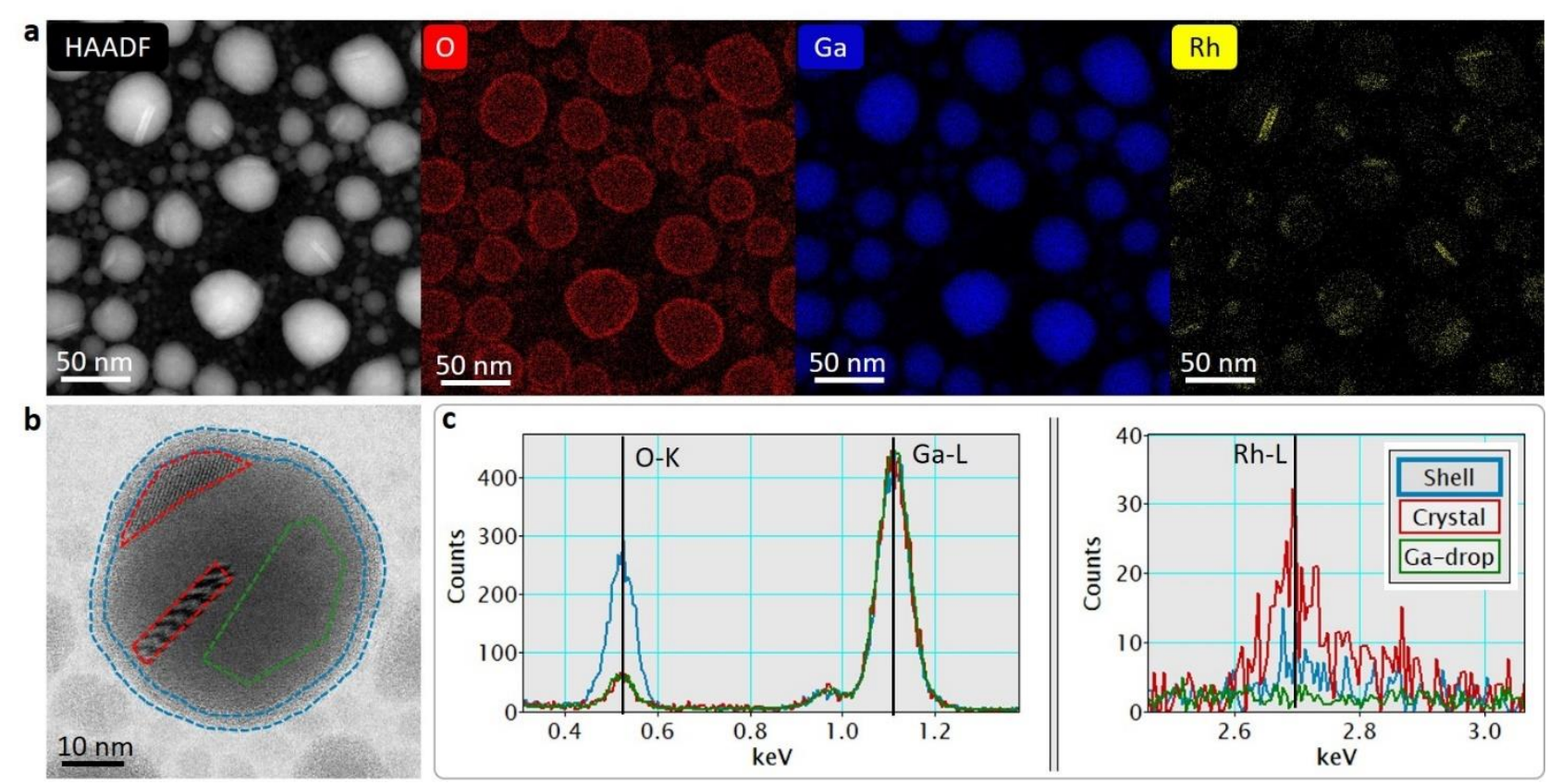

Figure 4: STEM-EDX analysis of a sample with an average Rh concentration of 1.8 at. \%, oxidized in atmospheric air for $\approx 2$ weeks. a) Simultaneously acquired STEM-HAADF (Z-contrast) image and the background-subtracted EDX signals of (from left to right) $O(O \mathrm{~K}), \mathrm{Ga}(\mathrm{Ga} \mathrm{L})$, and $R h(R h \mathrm{~L})$. The existence of an O-containing shell at the periphery of the particles is clearly visible in the $O E D X$ map (red). b) The STEM-BF image of a single particle shows a distinct amorphous contrast of $a \approx 4$ $\mathrm{nm}$ thick oxide shell. Lattice fringe contrast from the Rh-rich nanocrystal phase is marked by red dash lines. c) Area integrated EDX spectra of the oxide shell (blue), Rh-rich nanocrystals (red), and metallic Ga droplet (green). The intensity of the EDX spectra is normalized to the intensity of the Ga-L emission edge to account for the different number of pixels in the integral signals.

Figure 4 summarizes the findings for a sample with an average Rh concentration of 1.8 at. \% (as determined by EDX). The nanoparticles show a round shape (in projection) and a typical twophase microstructure of crystalline precipitates in an amorphous/liquid (a-) Ga matrix, similar to what we reported before.[4] Prior to the TEM studies, the sample was oxidized in atmospheric air for about 2 weeks after deposition. From the STEM-EDX maps in Figure 4a, an approximately $4 \mathrm{~nm}$ thick oxide shell is obvious. The bright field STEM image in Figure $4 \mathrm{~b}$ clearly shows a distinct amorphous shell (indicated by the blue dashed lines) between the darker Ga droplet (with some crystal precipitates, indicated in red) and the brighter membrane support. The crystalline precipitates appear at the $\mathrm{Ga} / \mathrm{Ga}_{2} \mathrm{O}_{3}$ interface. STEM-EDX mapping of this particle allows us to detect the spatial distribution of Rh, as shown in Figure 4c. In the crystallites (red), Rh shows the strongest signal, while it is not detectable in the amorphous/liquid Ga particle region (green). In the oxide shell (blue), a weak Rh signal is detected, in line with the surface Rh enrichment found in the XPS measurements after oxidation.

In-situ heating of the particles to $550^{\circ} \mathrm{C}(823 \mathrm{~K})$ results in the desorption of the oxide film. At even higher temperatures, the IMCs liquefy and recrystallize when cooled.[4] Figure 5 shows Ga- 
Rh alloy particles after heating, i.e., for which the oxide has been desorbed. Here again the Rh-rich c-Ga-Rh intermetallic phases are evident (encircled in red in Fig. 5b). It has to be noted that the TEM results have been obtained after cooling down to room temperature. Thus, precipitation of Rh-rich IMCs from the liquid phase is expected. A more detailed description of the microscopic results obtained for Rh-Ga SCALMS particles can be found elsewhere.[4]
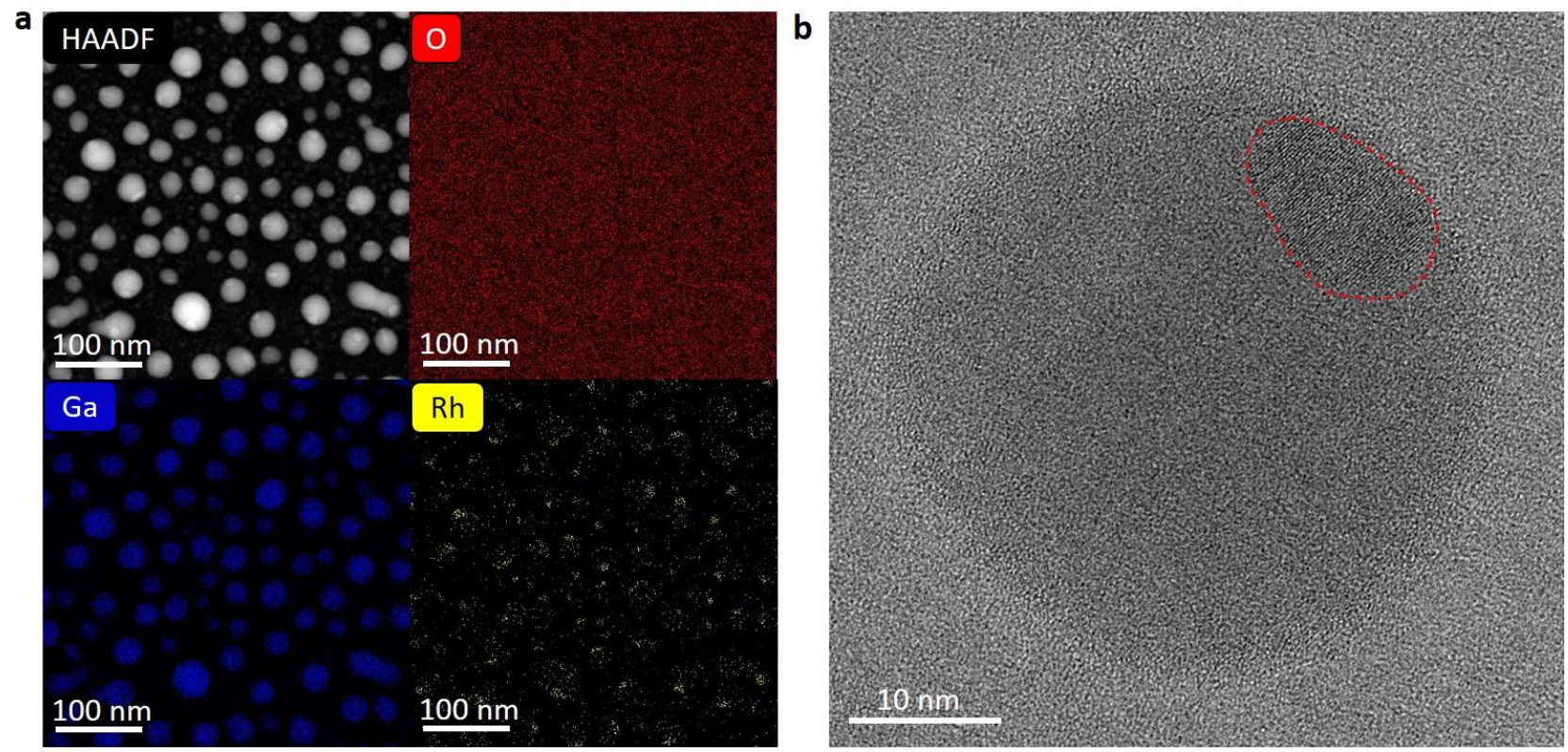

Figure 5. Microscopy data of a sample (measured at room temperature) after the oxide layer has been desorbed by heating to $550^{\circ} \mathrm{C}$ in vacuum in the TEM. (a) Simultaneous STEM-ADF and background subtracted STEM-EDX signal maps of $O(O \mathrm{~K}), \mathrm{Ga}(\mathrm{Ga} L)$, and $R h(R h \mathrm{~L})$. No more oxide shell is visible from the maps, and the homogeneous $O$ signal results from the thin $\mathrm{SiO}_{x}$ supporting membrane. (b) HRTEM image of a single particle. The lattice contrast as marked by the red dashed circle indicates a Ga-rich crystalline $G a_{1-x} R h_{x}$ phase (see SI of [4]), which appears to attach directly to the vacuum interface of the particle.

In a next step, we investigated a similar $125 \AA$ thick Rh-Ga model catalyst with 2.5 at.\% Rh, deposited on the native oxide film $\left(\mathrm{SiO}_{\mathrm{x}}\right)$ of a $\mathrm{Si}(111)$ wafer $\left(\mathrm{d}_{\text {siox: }} 10.1 \AA\right.$ according to XPS) using synchrotron-radiation-based XPS. The spectra were recorded with photon energies of 449 and $1045 \mathrm{eV}$, resulting in more surface- or more bulk-sensitive information on the nanoalloy particles, respectively. Note that significant carbon impurities in/on the $\mathrm{SiO}_{x} / \mathrm{Si}$-substrate were detected prior to deposition. Regarding the oxidation behavior, this system is comparable to the presented microscopy studies, despite the slightly higher Rh content. After preparation and initial characterization, the model catalyst was oxidized for $\approx 10 \mathrm{~min}$ at room temperature under ambient conditions in order to ensure comparability to the microscopy studies and then re-characterized. 
Figure 6 summarizes the recorded data. The upper two spectra in Figure 6a show the changes in the $\mathrm{Rh} 3 \mathrm{~d}$ region upon deposition of $\mathrm{Ga}$ onto the pure $\mathrm{Rh}$ particles on $\mathrm{SiO}_{\mathrm{x}}$, measured with a photon energy of $449 \mathrm{eV}$. The Rh $3 \mathrm{~d}$ doublet has a distinct, asymmetric shape with a $\mathrm{Rh} 3 \mathrm{~d}_{5 / 2}$ signal at $307.0 \mathrm{eV}$ and a FWHM of $0.84 \mathrm{eV}$. Upon Ga deposition, the Rh $3 \mathrm{~d}_{5 / 2}$ signal shifts to $307.6 \mathrm{eV}$, narrows to a FWHM of $0.52 \mathrm{eV}$, and becomes more symmetric. In contrast to the macroscopic alloy droplet, a significant $\mathrm{Rh} 3 \mathrm{~d}$ signal is found for the sequentially deposited Rh-Ga model catalyst at room temperature, even before oxidation. This observation is attributed to the finite size of the nanoparticles and the preferred formation of IMCs also at the vacuum interface of the formed nanoparticles (see Figure 4). The loss in peak asymmetry was also, to a lesser extent, observed for the macroscopic alloy droplets, see Figure 3.

All these observations agree with what is expected for the system: Upon deposition of $2.2 \AA \mathrm{Rh}$ on $\mathrm{SiO}_{x}$, small Rh particles form, leading to a $\mathrm{Rh} 3 \mathrm{~d}_{5 / 2}$ peak at $307.0 \mathrm{eV}$, which is shifted by $0.4 \mathrm{eV}$ to lower binding energy relative to the $\mathrm{Rh}(111)$ reference at $307.4 \mathrm{eV}$ (Fig. 3). This observation is in line with results for $\mathrm{Rh}$ particles on $\mathrm{SiO}_{\mathrm{x}}$, where a similar negative binding energy shift was attributed to interactions with the substrate.[43] In analogy to our observations for the macroscopic alloy droplet (see above), upon Ga deposition onto the Rh particles, the $\mathrm{Rh} 3 \mathrm{~d}_{5 / 2}$ signal shifts to $307.6 \mathrm{eV}$, which is identical to the binding energy observed for Rh-Ga intermetallic compounds. This is in line with the TEM results (see Figure 5), where intermetallic phases are shown to crystallize close to the vacuum/particle interface, i.e., within the detection volume probed by XPS. This situation is in contrast to the macroscopic alloy droplets, where the Rh-Ga intermetallic compound crystallites are mostly located in the bulk of the droplets or at the droplet/support interface and therefore cannot be probed by XPS. The observed narrowing of the signal and the loss in asymmetry is related to the change in particle size distribution and to the decrease in density of states close to the Fermi level when comparing pure Rh to the Rh-Ga system. It was already reported for Pd-Ga intermetallic compounds that a loss in density of states close to the valence band edge results in a reduction of the asymmetry observed in the Pd $3 d$ signals; the computational results discussed below show that similar changes in the valence band occur for the Rh-Ga system.[15, 21-23, 44]

The spectra at the bottom of Figure 6 show the changes in the Rh $3 \mathrm{~d}$ and $\mathrm{Ga} 3 \mathrm{~d}$ regions after exposing the $\mathrm{Rh}-\mathrm{Ga} / \mathrm{SiO}_{2}$ system to air. For the surface-sensitive $\mathrm{Rh}$ measurement with a photon energy of $449 \mathrm{eV}$ in Figure $6 \mathrm{a}$, the $\mathrm{Rh} 3 \mathrm{~d}_{5 / 2}$ peak at $307.6 \mathrm{eV}$ decreased to $39 \%$ of its previous intensity after oxidation. Furthermore, the signal broadens towards higher binding energy, which can be explained by an additional component shifted $0.4 \mathrm{eV}$ to higher binding energy from the main Rh signal. This binding energy of the additional component agrees with the results obtained from the oxidized macroscopic alloy droplet, where we assigned the component with highest binding energy to $\mathrm{Rh}$ atoms incorporated into $\mathrm{Ga}_{2} \mathrm{O}_{3}$. This observation, in combination with the 
HRTEM and spatially resolved EDX results, supports the interpretation of incorporated Rh into $\mathrm{Ga}_{2} \mathrm{O}_{3}$ leading to a changed $\mathrm{Rh} 3 \mathrm{~d}$ signal shape upon oxidation.

Figure $6 \mathrm{~b}$ shows the $\mathrm{Ga} 3 \mathrm{~d}$ region measured at photon energy $449 \mathrm{eV}$ before and after oxidation; here, the resolution is high enough to resolve the spin-orbit split $G a 3 d_{5 / 2}$ and $3 d_{3 / 2}$ doublet. The $\mathrm{Ga} 3 \mathrm{~d}_{5 / 2}$ signal is found at $18.4 \mathrm{eV}$. After oxidation, an additional broad peak appears shifted by $+2.0 \pm 0.2 \mathrm{eV}$ to higher binding energy relative to the metallic signals, which was fitted using two symmetric Voigt functions (blue). A further doublet (green) shifted by $\approx 1 \mathrm{eV}$ to higher binding energy from the metallic peak is needed to reproduce the measured signal shape. The binding energy of the latter has a larger margin of error, due to the larger FWHM and low intensity of the signal. A likely interpretation for this component is the $\mathrm{Ga} / \mathrm{Ga}_{2} \mathrm{O}_{3}$ interface, as suggested by Su et al., or the presence of small amounts of additional substoichiometric oxides (e.g. $\mathrm{Ga}_{2} \mathrm{O}$ ).[39, 40] 

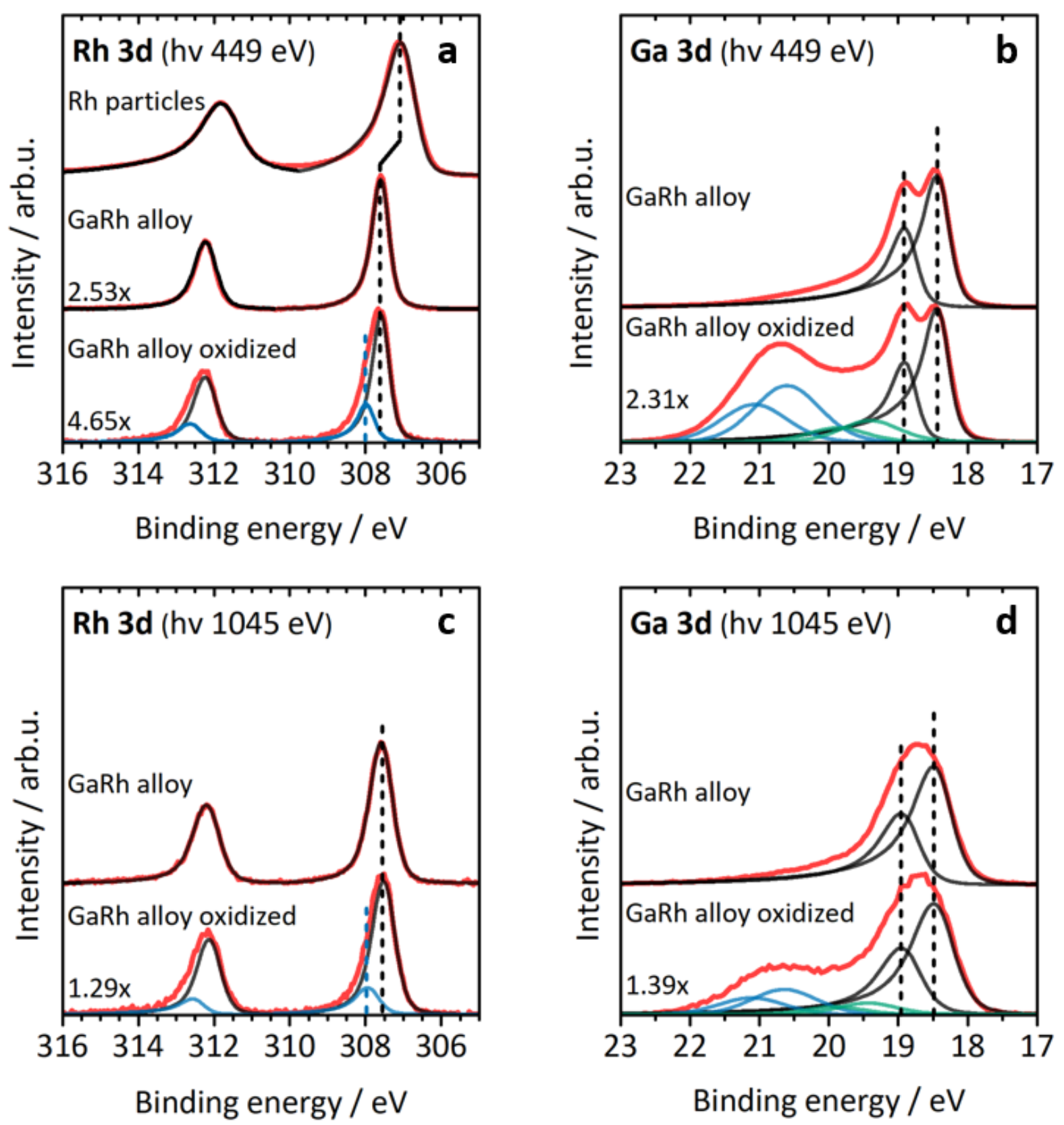

Figure 6: $(a, c) R h 3 d_{5 / 2}$ and $(b, d)$ Ga $3 d$ spectra of the Rh-Ga particle systems before (top spectra) and after oxidation (bottom spectra) probed with a photon energy of (a, b) 449 and (c, d) $1045 \mathrm{eV}$. The additional third spectrum at the top in panel (a) shows pure Rh particles before Ga deposition. Components considered metallic are black; components that appear after oxidation are blue and green. The spectra were height normalized for display. Scaling factors are given with respect to the first (top) spectrum in the subset.

Figure $6 \mathrm{c}$ and $6 \mathrm{~d}$ display the Rh $3 \mathrm{~d}$ and Ga $3 \mathrm{~d}$ spectra, respectively, measured with the higher photon energy of $1045 \mathrm{eV}$. The spectra show no differences in the binding energies of the observed components compared to the data in Figures $6 \mathrm{a}$ and $6 \mathrm{~b}$ probed by $449 \mathrm{eV}$ photon 
energy. The observed larger FWHMs at the higher photon energy are due to the lower energy resolution at higher photon energies (see Experimental). The different relative intensities of different features measured with two different photon energies originate from the larger information depth of $\approx 43 \AA$ ( $54 \AA$ ) for the $\mathrm{Rh} 3 \mathrm{~d}$ ( $\mathrm{Ga} 3 \mathrm{~d}$ ) electrons in $\mathrm{Ga}_{2} \mathrm{O}_{3}$ at $1045 \mathrm{eV}$ photon energy, as compared to $\approx 16 \AA$ ( $29 \AA$ ) for the $\mathrm{Rh} 3 \mathrm{~d}$ (Ga $3 \mathrm{~d}$ ) at $449 \mathrm{eV}$ photon energy. This effect is most pronounced in the area ratio of the oxidic to the metallic component of the $\mathrm{Ga} 3 \mathrm{~d}$ signal: at a photon energy of $449 \mathrm{eV}$ the ratio is 0.67 , whereas at $1045 \mathrm{eV}$ it is only 0.27 . The larger ratio of the oxidic to the metallic component obtained in the more surface-sensitive $449 \mathrm{eV}$ measurements further supports our interpretation that the oxide is formed as a closed wetting film on top of the metallic nanoalloy.

Furthermore, the ratio of the newly formed Rh $3 d_{5 / 2}$ component at $308.0 \mathrm{eV}$ to the $R \mathrm{~h} 3 \mathrm{~d}_{5 / 2}$ component at $307.6 \mathrm{eV}$ is larger at $449 \mathrm{eV}$ than at $1045 \mathrm{eV}$ photon energy (0.34 vs 0.26). The larger value obtained from the more surface-sensitive measurements indicates that the newly formed Rh component is located near the surface. This conclusion is in line with the aboveproposed $\mathrm{Rh}$ incorporation into the near surface of the oxide film. The following section on computational models for the $\mathrm{Ga}_{2} \mathrm{O}_{3} / \mathrm{Rh}-\mathrm{Ga}$ interface will examine the incorporation of $\mathrm{Rh}$ into $\mathrm{Ga}_{2} \mathrm{O}_{3}$ on an atomistic level.

\section{Computational results}

To obtain further insight, we investigated the systems described above by periodic DFT calculations using slab models. For Rh incorporation in $\mathrm{Ga}_{2} \mathrm{O}_{3}$, we considered two Rh sites, one tetrahedral and one octahedral, which are indicated in Figure $7 \mathrm{~b}$. Furthermore, the orthorhombic intermetallic compounds $\mathrm{Ga}_{16} \mathrm{Rh}_{3}$ and $\mathrm{Ga}_{21} \mathrm{Rh}_{4}$ were studied; see Figure 7c. [45] To access the liquid $\mathrm{Ga} / \mathrm{Ga}_{2} \mathrm{O}_{3}$ interface, we used three model systems, $\mathrm{A} 1$ to $\mathrm{A} 3$, that were constructed by combining AIMD simulations and geometry optimizations and that allow us to model both the liquid and solid environment present in the same slab, see Figure $7 \mathrm{~d}$. Details concerning the construction of the models are discussed in the $\mathrm{Sl}$, along with the cell dimensions and corresponding k-point meshes. Lattice constants were taken from experimental data. Using these structures, we will now discuss the stability and calculated core level shifts. To estimate the accuracy of our computational approach, we calculated the Ga $2 p$ and Ga $3 d$ core level energies in oxidized and non-oxidized systems. Our results, which are described in the $\mathrm{SI}$, show that the systems can be described by our DFT and support the formation of $\beta-\mathrm{Ga}_{2} \mathrm{O}_{3}$-like structures on the liquid Ga.

To confirm the claim that the newly developing Rh 3d peaks in the XP spectra during oxidation stem from the Rh-Ga IMCs (307.6 eV, relative to Rh(111): $+0.2 \mathrm{eV}$ ) and Rh incorporated in the oxide film (308.0 eV, relative to $\mathrm{Rh}(111):+0.6 \mathrm{eV}$ ), we calculated the corresponding core level shifts for the IMCs, and for three different models for the liquid $\mathrm{Ga} / \mathrm{\beta}_{-}-\mathrm{Ga}_{2} \mathrm{O}_{3}$ interface (A1-A3). The 
latter were obtained by replacing single Ga atoms at various sites (as shown in Figure 7d) by Rh atoms with subsequent optimization of the geometry. To get an idea of the thermodynamic stability of $\mathrm{Rh}$ in $\mathrm{\beta}-\mathrm{Ga}_{2} \mathrm{O}_{3}$ compared to $\mathrm{Rh}$ in metallic $\mathrm{Ga}$, we compared the energies of the model systems $A 1$ to $A 3$. Our calculations show that systems where Rh is surrounded only by $G a$ in the metallic part are much more stable (by over $4 \mathrm{eV}$ ) than systems with $\mathrm{Rh}$ in the oxidic part. The corresponding DFT energies relative to Rh in metallic Ga are shown in Table S5. These results lead us to the conclusion that, thermodynamically, Rh should more likely be dissolved in the liquid Ga than incorporated in the oxide. However, the experiments clearly show the inclusion of Rh in the oxide, and we therefore must conclude that this is a kinetic rather than a thermodynamic effect. Another observation from the energetic point of view is that Rh which is octahedrally surrounded by oxygen atoms is more stable (by at least $1.6 \mathrm{eV}$ in all approaches, see Table S5) than Rh in tetrahedral oxygen coordination. To investigate this effect in more detail, we calculated the stability of $\mathrm{Rh}$ in octahedral and tetrahedral sites of bulk $\beta-\mathrm{Ga}_{2} \mathrm{O}_{3}$ by replacing one $\mathrm{Ga}$ atom in a $\beta$ $\mathrm{Ga}_{2} \mathrm{O}_{3}$ bulk supercell by Rh. Using the PBE functional, we find that incorporation of $\mathrm{Rh}$ in an octahedral site is more stable, by $2.15 \mathrm{eV}$, than is the tetrahedral site. As PBE is known to fail e.g. in terms of exactly predicting band gaps and lattice constants for $\beta-\mathrm{Ga}_{2} \mathrm{O}_{3}$, we additionally tested the HSEO6 functional, which represents both band gaps and geometric structure quite well. We again find that the octahedral inclusion of $\mathrm{Rh}$ in the $\beta-\mathrm{Ga}_{2} \mathrm{O}_{3}$ lattice is more stable (by $2.50 \mathrm{eV}$ ) compared to the tetrahedral one.

The results for the $\mathrm{Rh} 3 \mathrm{~d}$ core level shift calculations are summarized in Table 1. Comparing the shifts for the IMCs with $\mathrm{Rh}(111)$ as a reference, it gets obvious that a qualitative agreement with experiments can be obtained, even though the shift is considerably overestimated in the calculations ( +0.95 and $+0.89 \mathrm{eV}$ for $\mathrm{Ga}_{16} \mathrm{Rh}_{3}$ and $\mathrm{Ga}_{21} \mathrm{Rh}_{4}$ (DFT) compared to $+0.2 \mathrm{eV}$ in the experiment). The positive core level shifts of the intermetallic compounds compared to $\mathrm{Rh}(111)$ is counterintuitive at first. According to the electronegativity values of 1.81 and 2.28 for Ga and Rh, respectively, Rh should be negatively charged in the intermetallic compounds, resulting in $\mathrm{Rh}$ states shifted to lower binding energies. To verify this, we calculated Bader charges, which are shown in the SI, Table S4. Indeed, the calculations show that Rh is negatively charged by around 0.9 e in both IMCs. Similar observations were made for Pd-Ga IMCs, where a negative charge was found on the Pd in quantum chemical calculations, but XPS showed a shift to higher binding energies compared to elemental Pd.[15, 22, 44] The authors of these studies explain the effect by a higher filling of the valence $d$-band, which alters the final state screening of the core hole and shifts the $\mathrm{Pd} 3 \mathrm{~d}$ signal to higher binding energies compared to $\mathrm{Pd}^{0}$. To check whether this is also the case for the Rh-Ga IMCs studied here, we calculated density of states (DOS) for $\mathrm{Ga}_{16} \mathrm{Rh}_{3}$, $\mathrm{Ga}_{21} \mathrm{Rh}_{4}$, and elemental $\mathrm{Rh}$ (shown in Figure 8). It is obvious that the DOS of the IMCs is reduced at the Fermi level compared to elemental Rh. The interaction between Ga and Rh shifts the d-band away from the Fermi-level towards lower energies. This modified electronic structure leads to the change in the core-hole screening and a positive shift in the core level binding energy. 
The binding energies for single $\mathrm{Rh}$ atoms in liquid $\mathrm{Ga}$ are shifted to slightly larger values (on average: $+0.38 \mathrm{eV}$ ), which is not in agreement with the experiment. However, if the core level shifts of the IMCs are referenced to Rh in liquid Ga instead of pure Rh, then the calculated values $(+0.57$ and $+0.51 \mathrm{eV})$ agree very well with the experimental value in Figure $3(307.6-307.1 \mathrm{eV}=$ $+0.5 \mathrm{eV}$ ). The reason for this might be a different level of accuracy in the description of pure $\mathrm{Rh}$ and Rh-Ga interactions in DFT, or to temperature and structure effects in the experiments that are not covered in the calculations.

\begin{tabular}{|c|c|c|c|c|c|c|}
\hline System & \multicolumn{3}{|c|}{ pure Rh } & \multicolumn{3}{|c|}{$\begin{array}{c}\text { Rh in } \\
\text { liquid Ga }\end{array}$} \\
\hline (1) Rh atoms in liquid Ga & \multicolumn{3}{|c|}{+0.38} & \multicolumn{3}{|c|}{0.00} \\
\hline $\mathrm{Ga}_{16} \mathrm{Rh}_{3}$ & \multicolumn{3}{|c|}{+0.95} & \multicolumn{3}{|c|}{+0.57} \\
\hline $\mathrm{Ga}_{21} \mathrm{Rh}_{4}$ & \multicolumn{3}{|c|}{+0.89} & \multicolumn{3}{|c|}{+0.51} \\
\hline & A1 & A2 & A3 & A1 & A2 & A3 \\
\hline (2) Metallic - Surface & +0.38 & +0.30 & +0.31 & 0.00 & -0.08 & -0.06 \\
\hline (3) 10 - Interface & l & +0.24 & +0.22 & I & -0.14 & -0.16 \\
\hline (4) 20 - Interface & -0.29 & +0.01 & -0.07 & -0.67 & -0.36 & -0.45 \\
\hline (5) 30 - Interface & / & +0.09 & -0.31 & / & -0.28 & -0.69 \\
\hline (6) 40 - Interface & -0.23 & -1.39 & -0.86 & -0.61 & -1.77 & -1.24 \\
\hline (7) 50 - Interface & / & +0.01 & -0.22 & / & -0.37 & -0.60 \\
\hline (8) $60-$ Interface & +1.34 & I & 1 & +0.96 & I & l \\
\hline (9) 40 - Bulk & -0.05 & -0.87 & -0.78 & -0.43 & -1.24 & -1.16 \\
\hline (10) 60 - Bulk & +1.39 & +1.14 & +0.60 & +1.01 & +0.76 & +0.23 \\
\hline (11) 40 - Surface & +1.19 & +0.98 & +0.98 & +0.81 & +0.60 & +0.61 \\
\hline (12) 50 - Surface & +1.13 & -0.03 & +0.61 & +0.76 & -0.40 & +0.23 \\
\hline
\end{tabular}

Table 1: DFT results for $\mathrm{Rh} 3 \mathrm{~d}$ core level shifts for $\mathrm{Rh}$ in liquid Ga, the IMCs as well as $\mathrm{A} 1$ to $\mathrm{A} 3$. In the first column the systems are specified. The next three columns show core level shifts with pure $\mathrm{Rh}$ as reference, while the last three columns have $\mathrm{Rh}$ in liquid $\mathrm{Ga}$ as reference.

Because the core level shifts calculated for mixed Rh-Ga systems seem to agree with the experiment, the remaining discussion about the core level shifts of $\mathrm{Rh}$ incorporated in $\mathrm{Ga}_{2} \mathrm{O}_{3}$ will be based on the values with $\mathrm{Rh}$ in liquid $\mathrm{Ga}$ as reference (last three columns in Table 1). The experiments revealed that $\mathrm{Rh}$ incorporated in the oxide is shifted to even higher binding energies by $+0.4 \mathrm{eV}(308.0 \mathrm{eV})$ compared to the solid IMCs $(307.6 \mathrm{eV})$ and by $+0.9 \mathrm{eV}$ compared to Rh in liquid Ga $(307.1 \mathrm{eV})$. We can find this behavior in our calculations only for systems where Rh occupies octahedral sites (systems (8) and (10) in Table 1) or is located close to the surface with enough oxygen atoms in close proximity (systems (11) and (12) in Table 1). All other cases yield smaller, mostly even negative, shifts compared to Rh in liquid Ga. This is a strong sign that Rh is indeed incorporated in the oxide film, but only at sites where it is octahedrally coordinated by 6 
oxygen atoms. Surprisingly, even occupation of the tetrahedral sites (systems (6) and (9) in Table 1), which in case of the Ga $2 p$ and Ga $3 d$ core levels results in very similar values as the octahedral sites, yields shifts to smaller binding energies. Additionally, it is possible to conclude that $\mathrm{Rh}$ is not enriched at the liquid $\mathrm{Ga}-\mathrm{Ga}_{2} \mathrm{O}_{3}$ interface, but rather in the bulk of the oxide film where this octahedral coordination environment is possible. Further discussions about the incorporation of $\mathrm{Rh}$ in the oxide film and differences between approaches A1-A3 can be found in the SI. In conclusion, our calculations are in line with the experimental results and explain them in more detail.
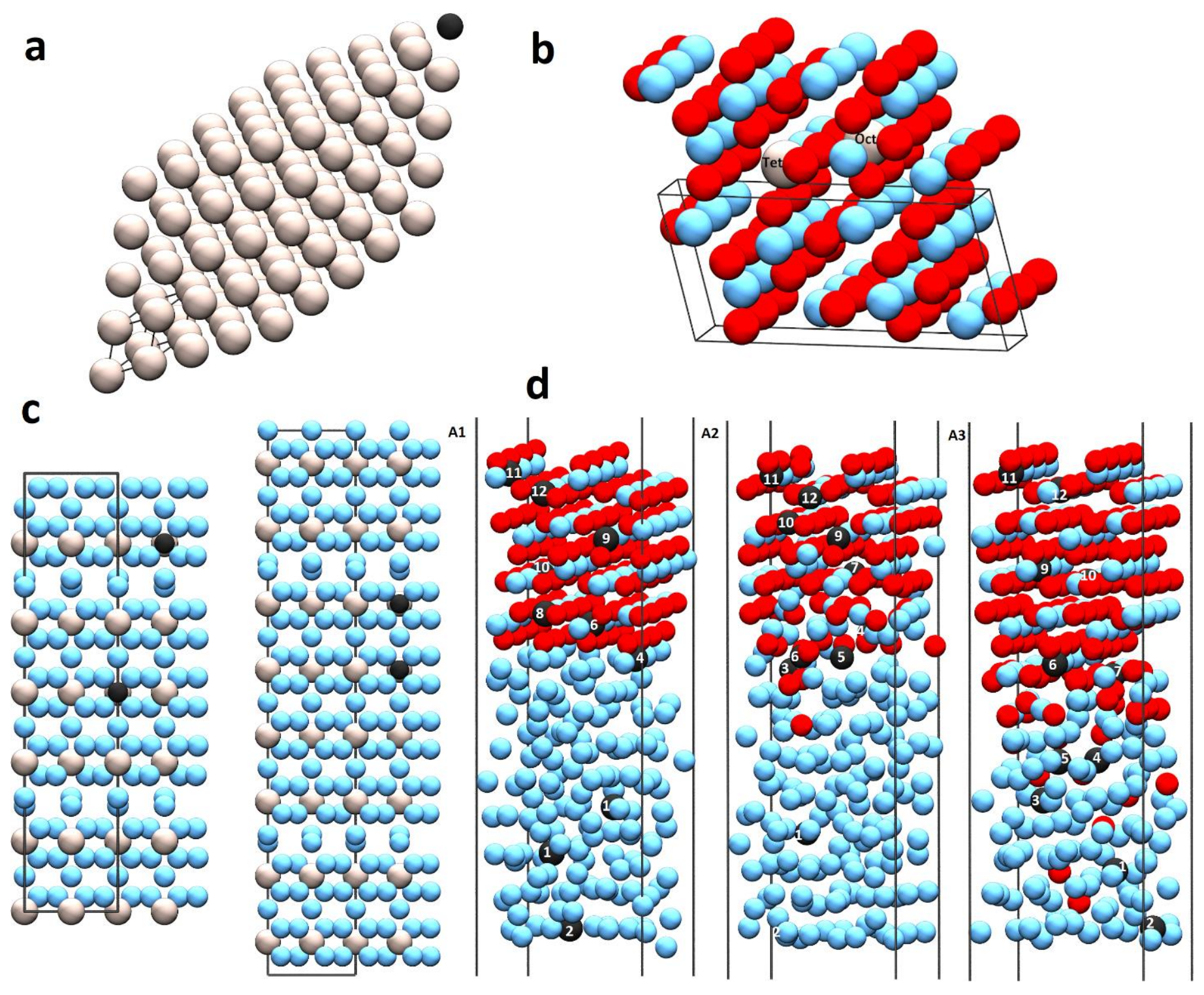

Figure 7: Systems that were investigated by DFT calculations. Ga is shown in light blue or black, oxygen in red, and Rh in silver and black. Black is used for those Rh atoms that were chosen for calculation of the core level binding energy. The unit cells are drawn with black lines, while the complete supercells that were used in the calculations are shown. a) fcc-Rh bulk system, b) $\beta$ - 
$\mathrm{Ga}_{2} \mathrm{O}_{3}$, c) $\mathrm{Ga}_{16} \mathrm{Rh}_{3}$ (left) and $\mathrm{Ga}_{21} \mathrm{Rh}_{4}$ (right) and d) approaches for the liquid $\mathrm{Ga} / \mathrm{\beta}_{-} \mathrm{Ga}_{2} \mathrm{O}_{3}$ interface: models $A 1, A 2$, and $A 3$ (from left to right). In c) tetrahedral and octahedral Ga sites are marked; in d) the numbers correspond to the numbers in Table 1 and characterize the chemical environment of the Rh atom. Note that the number (1) can be used more than once, because it describes atoms in metallic Ga bulk conditions. The black atoms correspond to $\mathrm{Ga}$ atoms after the first geometry optimization. Subsequently they were replaced by Rh and reoptimized, which is not shown here.
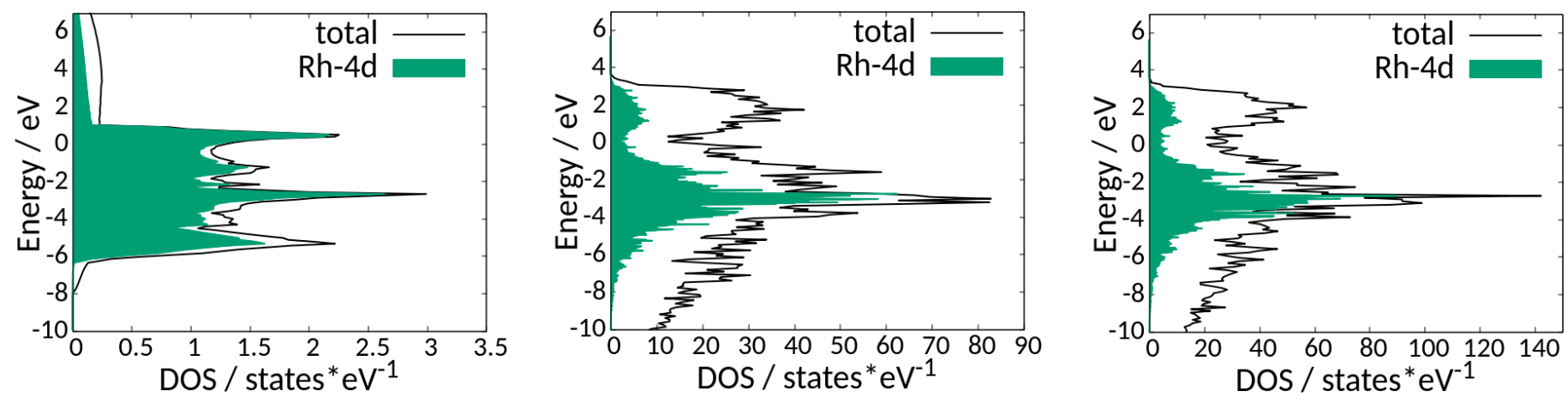

Figure 8: Total density of states (black) and Rh 4d DOS (green) for elemental Rh and the IMCs $\mathrm{Ga}_{16} \mathrm{Rh}_{3}$ and $\mathrm{Ga}_{21} \mathrm{Rh}_{4}$ (from left to right). 


\section{Conclusion}

To investigate the influence of molecular oxygen on Rh-Ga-based SCALMS catalysts and the properties of the $\mathrm{Ga}_{2} \mathrm{O}_{3} / \mathrm{Rh}-\mathrm{Ga}$ interface that is expected to form in the process; macroscopic $\mathrm{Rh}$ Ga samples were oxidized in situ in a lab-based near ambient pressure XPS system. Alongside the formation of a wetting $\mathrm{Ga}_{2} \mathrm{O}_{3}$ film on the surface of the alloy, a redistribution of the $\mathrm{Rh}$, resulting in a Rh surface enrichment, was observed. During the experiments, the $\mathrm{Ga}_{2} \mathrm{O}_{3}$ film thickness and surface compositions were monitored by near ambient pressure and UHV XPS. With increasing film thickness, no attenuation of the Rh signals was observed, leading to the conclusion that $\mathrm{Rh}$ is incorporated into the forming solid oxide film rather than remaining in the liquid alloy. A closer investigation of the Rh $3 \mathrm{~d}$ binding energies suggests that the enrichment results a) from Rh-Gabased intermetallic compounds and $b$ ) from $\mathrm{Rh}$ incorporated into the $\mathrm{Ga}_{2} \mathrm{O}_{3}$ film by substituting $\mathrm{Ga}$ in the atomic arrangement of the film. The oxidation process was found to depend on the oxygen pressure: for oxidation at higher pressures, the arising $\mathrm{Rh}$ signal results mostly from $\mathrm{Rh}$ incorporated into $\mathrm{Ga}_{2} \mathrm{O}_{3}$, while at lower pressures binding energies corresponding to intermetallic compounds are found.

Using a combination of AIMD and geometry optimization, we modeled a film of $\beta-\mathrm{Ga}_{2} \mathrm{O}_{3}$ on liquid $\mathrm{Ga}$ and calculated core level binding energies that showed agreement with experiments. The Rh, which is incorporated in the oxide film, is likely found substituting Ga cations at octahedrally coordinated lattice sites in the bulk oxide. The calculations also suggest that an incorporation of $\mathrm{Rh}$ into $\beta-\mathrm{Ga}_{2} \mathrm{O}_{3}$ is less favorable than Rh staying in bulk $\mathrm{Ga}$ solution. Therefore, the incorporation of $\mathrm{Rh}$ is most likely a kinetic effect. A rapid oxidation at higher pressures might result in an energetically unfavorable trapping of Rh atoms in the forming oxide film while at lower pressures $\mathrm{Rh}$ atoms retreat from the forming oxide. The higher local Rh concentration at the boundaries of the forming oxide layer could result in the formation of crystalline Rh-Ga phases at the $\mathrm{Ga} / \mathrm{Ga}_{2} \mathrm{O}_{3}$ interface.

To check transferability of the obtained results to more complex systems that closely resemble the real catalyst, $\mathrm{Rh}-\mathrm{Ga}$ particles were deposited on $\mathrm{SiO}_{2}$ substrates by physical vapor deposition. The morphology of the deposited particles was characterized by high resolution TEM, spatially resolved EDX, and synchrotron-based XPS after exposure to ambient. Microscopy showed a Rh enrichment in the $\mathrm{Ga}_{2} \mathrm{O}_{3}$ shell around the particles. No crystallites of Rh or Rh-Ga intermetallic compounds in the oxide shell are detected. Synchrotron-based XPS also showed the Rh enrichment in the oxide shell, attested to the same type incorporation of Rh atoms into the $\mathrm{Ga}_{2} \mathrm{O}_{3}$ structure as for the macroscopic samples. According to our current understanding, these results should be transferable to the real Rh-Ga based catalyst and similar effects are expected to occur upon exposing the real catalyst to molecular oxygen. Therefore, we consider these results as being highly relevant for the catalysis with SCALMS systems. 


\section{Supplementary Material}

See the supplementary material for further spectra of the oxidation $550 \mathrm{~K}$ together with fits and the quantitative analysis and data from the oxidation at $305 \mathrm{~K}$. This data is complemented by a description of the calculation of the thickness. Furthermore, we give more details on the computational models and results.

\section{Acknowledgement}

$\mathrm{JF}, \mathrm{RGW}$, and MB thank the whole EMIL commissioning team, especially M. Gorgoi and S. Hendel who made the beam available for these measurements.

\section{Data availability statement:}

The data that support the findings of this study are available from the corresponding author upon reasonable request.

\section{Literature}

1. Bauer, T. et al. Operando DRIFTS and DFT Study of Propane Dehydrogenation over Solid-and Liquid-Supported $\mathrm{Ga}_{x} \mathrm{Pt}_{\mathrm{y}}$ Catalysts. ACS Catal. 2019, 9, p. 2842-2853.

2. Görling, A.; Steinrück, H.-P.; Taccardi, N.; Wasserscheid, P.; Debuschewitz, J. Catalytically Active Compositions of Matter. Patent WO 2018036672 A1, 2018.

3. Grabau, M. et al. Spectroscopic Observation and Molecular Dynamics Simulation of Ga Surface Segregation in Liquid Pd-Ga Alloys. Chem. - Eur. J. 2017, 23, p. 17701-17706.

4. Raman, N. et al. Highly Effective Propane Dehydrogenation Using Ga-Rh Supported Catalytically Active Liquid Metal Solutions. ACS Catal. 2019, 9, p. 9499-9507.

5. Taccardi, N. et al. Gallium-rich Pd-Ga Phases as Supported Liquid Metal Catalysts. Nat. Chem. 2017, 9, p. 862-867.

6. Hohner, C. et al. Pt-Ga Model SCALMS on Modified HOPG: Thermal Behavior and Stability in UHV and under Near-Ambient Conditions. J. Phys. Chem. C. 2020, 124, 4, p.2562-2573.

7. Hohner, C. et al. Pt-Ga Model SCALMS on Modified HOPG: Growth and Adsorption Properties. Top. Catal. 2019, 62, p. 849-858.

8. Kettner, M. et al. Characterization and Stability of Pd Single Atom Sites. J. Catal. 2019, 369, 33-46.

9. Chabala, J.M. Oxide-Growth Kinetics and Fractal-Like Patterning across Liquid Gallium Surfaces. Phys. Rev. B 1992, 46, p. 11346-11357.

10. Regan, M.J. et al. Surface Layering in Liquid Gallium - an X-Ray Reflectivity Study. Phys. Rev. Lett. 1995, 75, p. 2498-2501.

11. Regan, M.J. et al. X-ray studies of atomic layering at liquid metal surfaces. J. Non-Cryst. Solids 1996, 207, p. 762-766.

12. Regan, M.J. et al. Capillary-wave roughening of surface-induced layering in liquid gallium. Phys. Rev. B 1996, 54, p. 9730-9733.

13. Regan, M.J. et al. X-ray study of the oxidation of liquid-gallium surfaces. Phys. Rev. B 1997, 55, p. 10786-10790.

14. Grabau, M. et al. Surface enrichment of $\mathrm{Pt}$ in $\mathrm{Ga}_{2} \mathrm{O}_{3}$ films grown on liquid $\mathrm{Pt} / \mathrm{Ga}$ alloys. Surf. Sci. 2016, 651, p. 16-21. 
15. Armbruster, M. et al. Intermetallic compounds in heterogeneous catalysis-a quickly developing field. Sci. Technol. Adv. Mater. 2014, 15, 034803.

16. Iwasa, N. et al. New catalytic functions of Pd-Zn, Pd-Ga, Pd-In, Pt-Zn, Pt-Ga and Pt-In alloys in the conversions of methanol. Catal. Lett. 1998, 54, p. 119-123.

17. Okamoto, H. Ga-Pd (Gallium-Palladium). J. Phase Equilib. Diffus. 2008, 29, p. 466-467.

18. Anres, P. et al. Thermodynamics of the (Rh-Ga) system. J. Alloys Compd. 1998, 265, p. 201-208.

19. Haghofer, A. et al. In situ study of the formation and stability of supported $\mathrm{Pd}_{2} \mathrm{Ga}$ methanol steam reforming catalysts. J. Catal. 2012, 286, p. 13-21.

20. Wowsnick, G. et al. Surface dynamics of the intermetallic catalyst $\mathrm{Pd}_{2} \mathrm{Ga}$, Part II - Reactivity and stability in liquid-phase hydrogenation of phenylacetylene. J. Catal. 2014, 309, p. 221-230.

21. Wowsnick, G. et al. Surface dynamics of the intermetallic catalyst $\mathrm{Pd}_{2} \mathrm{Ga}$, Part I - Structural stability in UHV and different gas atmospheres. J. Catal. 2014, 309, p. 209-220.

22. Kovnir, K. et al. A new approach to well-defined, stable and site-isolated catalysts. Sci. Technol. Adv. Mater. 2007, 8, p. 420-427.

23. Kovnir, K., et al., Pinning the catalytic centre: A new concept for catalyst development. Bessy Highlights. 2007, 2008, p. 22.

24. Pantforder, J. et al. New setup for in situ x-ray photoelectron spectroscopy from ultrahigh vacuum to 1 mbar. Rev. Sci. Instrum 2005, 76, 014102.

25. Jeurgens, L.P.H. et al. Composition and chemical state of the ions of aluminium-oxide films formed by thermal oxidation of aluminium. Surf. Sci. 2002, 506(3), p. 313-332.

26. Tanuma, S. et al. Calculation of electron inelastic mean free paths (IMFPs) VII. Reliability of the TPP2M IMFP predictive equation. Surf. Interface Anal. 2003, 35, p. 268-275.

27. Kresse, G. et al. Efficient iterative schemes for ab initio total-energy calculations using a plane-wave basis set. Phys. Rev. B 1996, 54(16), p. 11169-11186.

28. Kresse, G. et al. Efficiency of ab-initio total energy calculations for metals and semiconductors using a plane-wave basis set. Comput. Mater. Sci. 1996, 6(1), p. 15-50.

29. Kresse, G. et al. From ultrasoft pseudopotentials to the projector augmented-wave method. Phys. Rev. B 1999, 59(3), p. 1758-1775.

30. Perdew, J.P. et al. Generalized gradient approximation made simple. Phys. Rev. Lett. 1996, 77(18), p. 3865-3868.

31. Heyd, J. et al. Hybrid functionals based on a screened Coulomb potential. J. Chem. Phys. 2003, 118(18), p. 8207-8215.

32. Mermin, N.D. et al. Thermal Properties of the Inhomogeneous Electron Gas. Phys. Rev. 1965, 137(5A), p. A1441-A1443.

33. Nose, S. et al. A Unified Formulation of the Constant Temperature Molecular-Dynamics Methods. J. chem. phys. 1984, 81(1): p. 511-519.

34. Methfessel, M. et al. High-precision sampling for Brillouin-zone integration in metals. Phys. Rev. B 1989, 40(6): p. 3616-3621.

35. Henkelman, G. et al. A fast and robust algorithm for Bader decomposition of charge density. Comput. Mater. Sci. 2006, 36(3): p. 354-360.

36. Tang, W. et al. A grid-based Bader analysis algorithm without lattice bias. J. Phys. Condens. Matter 2009, 21(8).

37. Carli, R. et al. XPS Analysis of Gallium Oxides. Appl. Surf. Sci. 1994, 74, p. 99-102.

38. Schön, G. et al. Auger and direct electron spectra in X-ray photoelectron studies of zinc, zinc oxide, gallium and gallium oxide. J. ELECTRON SPECTROSC. 1973, 2(1), p. 75-86.

39. Su, C.Y. et al. Photoemission-Studies of Room-Temperature Oxidized Ga Surfaces. Surf. Sci. 1982, $118(1-2)$, p. 248-256. 
40. Surdu-Bob, C.C. et al. An X-ray photoelectron spectroscopy study of the oxides of GaAs. appl. surf. sci. 2001, 183(1-2), p. 126-136.

41. Kibis, L.S. et al. XPS Study of Nanostructured Rhodium Oxide Film Comprising $\mathrm{Rh}^{4+}$ Species. J. Phys. Chem. C 2016, 120(34), p. 19142-19150.

42. Moulder, J.F. et al. Handbook of X-ray Photoelectron Spectroscopy: A Reference Book of Standard Spectra for Identification and Interpretation of XPS Data 1992, Physical Electronics Division, PerkinElmer Corporation.

43. Lindner, U. et al. XPS and TEM Characterization of Pure and Th, Pr or Ce Oxide Containing Rh/SiO Catalysts. Fresenius J. Anal. Chem. 1991., 341(5-6), p. 387-394.

44. Kovnir, K. et al. In situ surface characterization of the intermetallic compound PdGa - A highly selective hydrogenation catalyst. Surf. Sci. 2009, 603(10-12), p. 1784-1792.

45. Bostrom, M. et al. Preparation, crystal structure and chemical bonding analysis of the new binary compounds $\mathrm{Rh}_{4} \mathrm{Ga}_{21}$ and $\mathrm{Rh}_{3} \mathrm{Ga}_{16}$. J. Solid State Chem. 2006, 179(8), p. 2472-2478. 

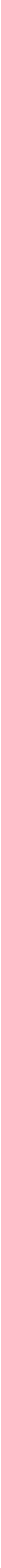

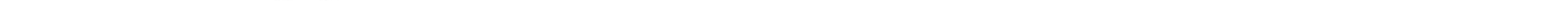


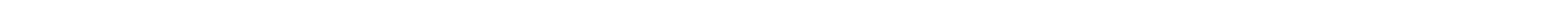




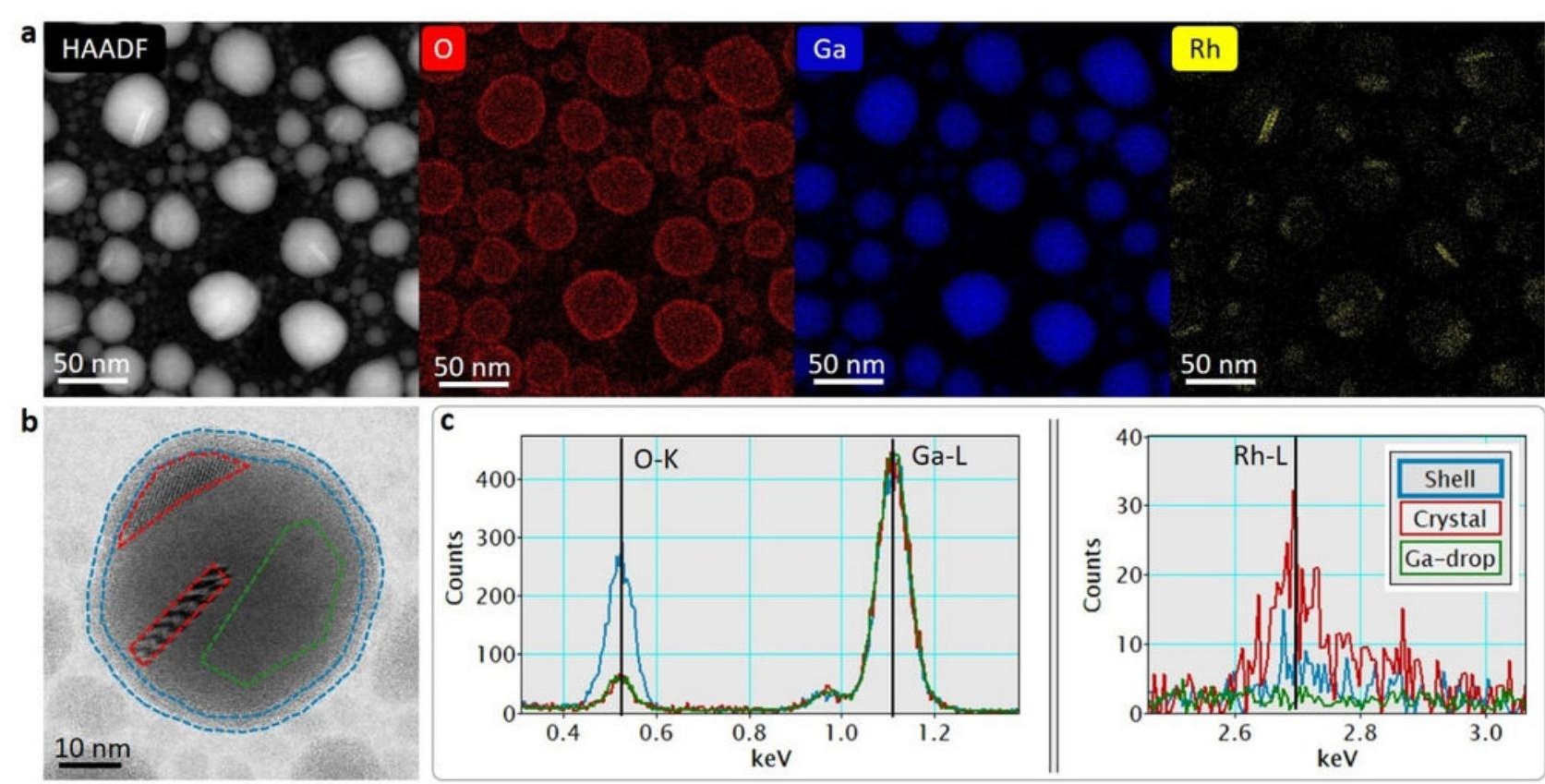



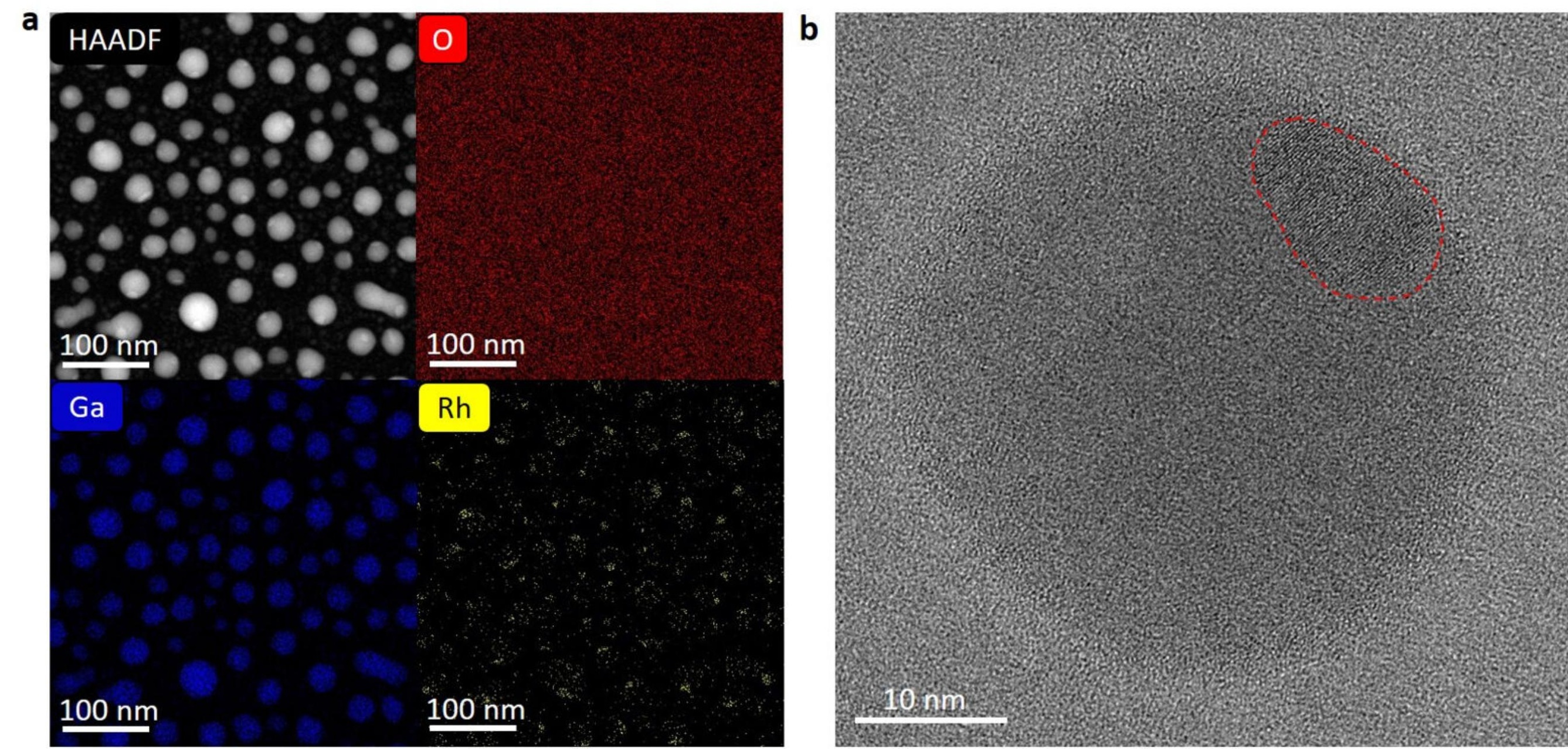
C

WO OO or yos torese $6 \%$ \& inu una Tocoloseo Y u Tos racosasos Tovo yoo toserase \& 0 \& 0 lar arou Locosos 100 yon toscososo Yo sooso roloros $48 \%$ yolonos tosoroso Y TNo Noreo yoscosos - ju Touroso Tosososo $\checkmark 40$ TouTsoo Tos \& 0 \& 0 you aros coscoso Y 000 coscos d

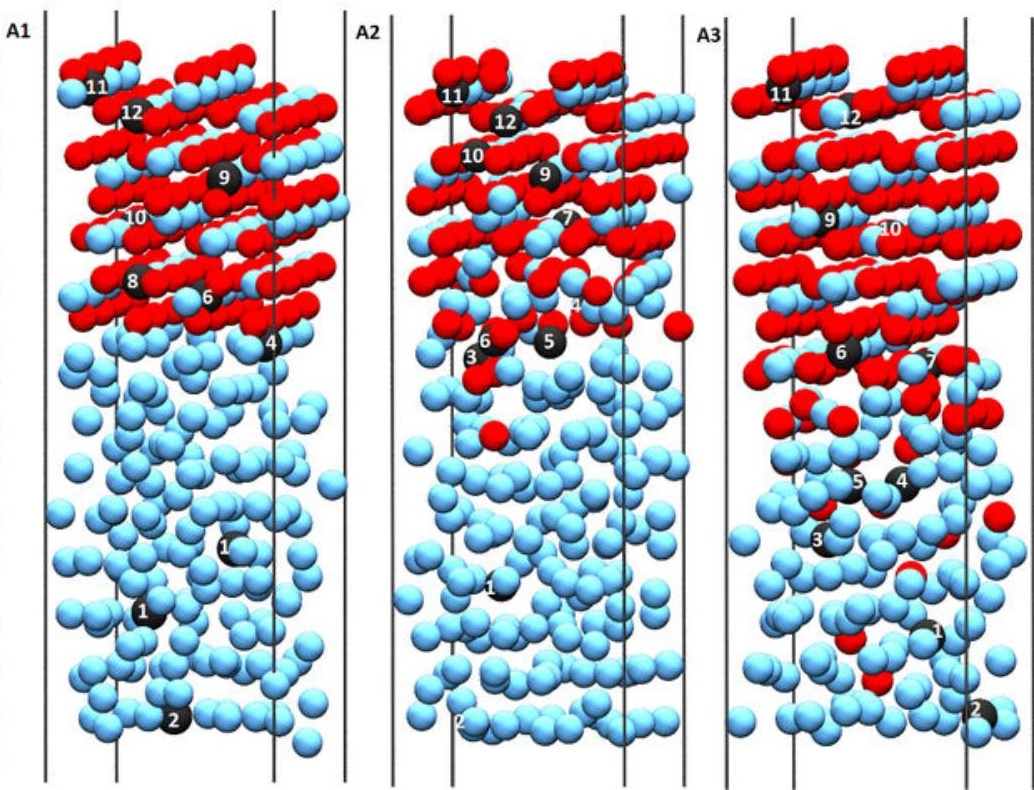


